\title{
CONSTANT SIGN AND NODAL SOLUTIONS FOR NONLINEAR ELLIPTIC EQUATIONS WITH COMBINED NONLINEARITIES*
}

\author{
SERGIU AIZICOVICI ${ }^{\dagger}$, NIKOLAOS S. PAPAGEORGIOU ${ }^{\ddagger}$, AND VASILE STAICU $§$
}

\begin{abstract}
We study a parametric nonlinear Dirichlet problem driven by a nonhomogeneous differential operator and with a reaction which is "concave" (i.e., $(p-1)$ - sublinear) near zero and "convex" (i.e., $(p-1)$ - superlinear) near $\pm \infty$. Using variational methods combined with truncation and comparison techniques, we show that for all small values of the parameter $\lambda>0$, the problem has at least five nontrivial smooth solutions (four of constant sign and the fifth nodal). In the Hilbert space case $(p=2)$, using Morse theory, we produce a sixth nontrivial smooth solution but we do not determine its sign.
\end{abstract}

Key words. Nodal solutions, nonlinear regularity, local minimizer, extremal solutions, critical groups, superlinear reaction, concave term.

AMS subject classifications. 35J20, 35J60, 35J92, 58E05.

1. Introduction. In this paper, we study the following nonlinear boundary value problem

$$
-\operatorname{div} a(z, D u(z))=f(z, u(z), \lambda) \text { in } \Omega,\left.u\right|_{\partial \Omega}=0 .
$$

Here $\Omega \subseteq \mathbb{R}^{N}$ is a bounded domain with a $C^{2}$ - boundary $\partial \Omega, a: \bar{\Omega} \times \mathbb{R}^{N} \rightarrow \mathbb{R}^{N}$ is a continuous map which is $C^{1}$ on $\bar{\Omega} \times \mathbb{R}^{N} \backslash\{0\}$ and satisfies certain other regularity conditions (see hypotheses $\left.\left(H_{0}\right)\right)$ and $f: \Omega \times \mathbb{R} \times(0,+\infty) \rightarrow \mathbb{R}$ is a reaction term which is Carathéodory in the $(z, x) \in \Omega \times \mathbb{R}$ variables (i.e., for all $x \in \mathbb{R}, z \rightarrow f(z, x, \lambda)$ is measurable, and for almost all $z \in \Omega, x \rightarrow f(z, x, \lambda)$ is continuous) and $\lambda>0$ is a parameter. We mention that a special case of the differential operator is the $p$-Laplacian differential operator. However, we stress that in contrast with the $p$-Laplacian, the differential operator in $\left(P_{\lambda}\right)$ needs not to be homogeneous. Concerning the reaction $f(z, x, \lambda)$, we assume that $x \rightarrow f(z, x, \lambda)$ exhibits $(p-1)$ - superlinear growth near $\pm \infty$, but we do not assume the usual in such cases Ambrosetti-Rabinowitz condition (AR-conditon, for short). Instead, we employ an alternative weaker condition, which incorporates in our framework functions with "slower" growth near $\pm \infty$. In addition, our hypotheses on $x \rightarrow f(z, x, \lambda)$ imply the presence of "concave" $((p-1)-$ sublinear) terms in the reaction. So, on the right hand side of $\left(P_{\lambda}\right)$ we have the combined effects of "convex" and "concave" nonlinearities (competition phenomena). A special case is the classical "convex-concave" nonlinearity of the form

$$
\lambda|x|^{q-2} x+|x|^{r-2} x \text { for all } x \in \mathbb{R}, \text { with } 1<q<p<r<p^{*},
$$

where

$$
p^{*}:=\left\{\begin{array}{cc}
\frac{N p}{N-p} & \text { if } p<N \\
+\infty & \text { if } p \geq N
\end{array}\right.
$$

\footnotetext{
* Received May 13, 2013; accepted for publication December 2, 2014.

$\dagger$ Department of Mathematics, Ohio University, Athens, OH 45701, USA (aizicovs@ohio.edu).

$\ddagger$ Department of Mathematics, National Technical University, Zografou Campus, Athens 15780, Greece (npapg@math.ntua.gr).

$\S$ Department of Mathematics, CIDMA, University of Aveiro, Campus Universitário de Santiago, 3810-193 Aveiro, Portugal (vasile@ua.pt).
} 
Such equations with combined (competing) nonlinearities were first investigated in the context of Dirichlet equations driven by the Laplacian, by Ambrosetti-Brezis-Cerami [6] and subsequently by Li-Wu-Zhou [27]. These semilinear works were extended to equations driven by the $p$ - Laplacian by Garcia Azorero-Manfredi-Peral Alonso [19], Guo-Zhang [21], Hu-Papageorgiou [23] and Kyritsi-Papageorgiou [25]. In the aforementioned works, the authors either consider only the existence and multiplicity of positive solutions or prove multiplicity results without providing sign information for all the solutions. We should also mention the very recent work of AizicoviciPapageorgiou-Staicu [4], which is also concerned with equations driven by a nonhomogeneous differential operator and include a $(p-1)$ - superlinear reaction. However, the hypotheses of [4] preclude the presence of concave terms near the origin (no competition phenomena) and the main multiplicity theorem does not provide sign information for all the solutions. Finally, a comparable study of a more restrictive class of nonlinear parametric periodic problems was recently conducted by the authors in $[5]$.

The aim of this work is to prove a multiplicity theorem for problem $\left(P_{\lambda}\right)$, with sign information for all the solutions. In particular, we look explicitly for nodal (i.e., sign changing) solutions. We show that there exists a critical parameter value $\lambda^{*}>0$ such that for all $\lambda \in\left(0, \lambda^{*}\right)$ problem $\left(P_{\lambda}\right)$ has at least five nontrivial smooth solutions, four of constant sign (two positive, the other two negative) and the fifth nodal. Note that the lack of homogeneity in the differential operator creates serious technical difficulties and the usual methods to produce nodal solutions fail (see, for example, Aizicovici-Papageorgiou-Staicu [2]). So, new techniques are needed in order to overcome these obstacles. At the end, we treat the Hilbert space case (i.e., $p=2$ ).

In particular, our results extend and complement the conclusions of the recent work of Marano-Papageorgiou [29], where a related problem driven by the p-Laplacian is considered. There, a different substitute of the AR-condition is used (see hypothesis $\left(f_{4}\right)$ ), and the method of proof depends heavily on the homogeneity of the p-Laplacian.

Our approach uses variational methods based on the critical point theory and Morse theory. In the next section, for easy reference, we recall some of the main mathematical tools that will be used in the sequel.

2. Mathematical background. Let $(X,\|\cdot\|)$ be a Banach space and $\left(X^{*},\|\cdot\|_{*}\right)$ be its topological dual. By $\langle.,$.$\rangle we denote the duality brackets for the pair \left(X^{*}, X\right)$. Let $\varphi \in C^{1}(X)$. A real number $c$ is said to be a critical value of $\varphi$ if there exists $x^{*} \in X$ such that $\varphi^{\prime}\left(x^{*}\right)=0$ and $\varphi\left(x^{*}\right)=c$.

We say that the functional $\varphi$ satisfies the Cerami condition (the C-condition for short), if the following is true:

every sequence $\left\{x_{n}\right\}_{n \geq 1} \subseteq X$ such that $\left\{\varphi\left(x_{n}\right)\right\}_{n \geq 1}$ is bounded in $\mathbb{R}$ and

$$
\left(1+\left\|x_{n}\right\|\right) \varphi^{\prime}\left(x_{n}\right) \rightarrow 0 \text { in } X^{*} \text { as } n \rightarrow \infty
$$

admits a strongly convergent subsequence.

This compactness-type condition is in general weaker than the usual "PalaisSmale condition" (the PS-condition for short). However, the C-condition suffices in order to obtain a deformation theorem and from it to derive the minimax theory for certain critical values of $\varphi \in C^{1}(X)$. In particular, we can state the following theorem, known in the literature as the "mountain pass theorem".

Theorem 1. If $\varphi \in C^{1}(X)$ satisfies the $C$-condition, $x_{0}, x_{1} \in X, \rho>$ $0,\left\|x_{1}-x_{0}\right\|>\rho, \max \left\{\varphi\left(x_{0}\right), \varphi\left(x_{1}\right)\right\}<\inf \left\{\varphi(x):\left\|x-x_{0}\right\|=\rho\right\}=\eta_{\rho}$, and 
$c=\inf _{\gamma \in \Gamma} \max _{t \in[0,1]} \varphi(\gamma(t))$, where $\Gamma=\left\{\gamma \in C([0,1], X): \gamma(0)=x_{0}, \gamma(1)=x_{1}\right\}$, then $c \geq \eta_{\rho}$ and $c$ is a critical value of $\varphi$.

In the last part of this paper, in order to distinguish between solutions and study the Hilbert space case (i.e., $p=2$ ), we will use critical groups. So, let us recall their definition. Let $X$ be a Banach space, $\varphi \in C^{1}(X)$ and $c \in \mathbb{R}$. We introduce the following sets

$$
\begin{aligned}
\varphi^{c} & =\{x \in X: \varphi(x) \leq c\}, \\
K_{\varphi} & =\left\{x \in X: \varphi^{\prime}(x)=0\right\}, \\
K_{\varphi}^{c} & =\left\{x \in K_{\varphi}: \varphi(x)=c\right\} .
\end{aligned}
$$

Let $\left(Y_{1}, Y_{2}\right)$ be a topological pair with $Y_{2} \subset Y_{1} \subset X$. For every integer $k \geq 0$, by $H_{k}\left(Y_{1}, Y_{2}\right)$ we denote the $k^{t h}$ - relative singular homology group of the pair $\left(Y_{1}, Y_{2}\right)$ with integer coefficients. The critical groups of $\varphi$ at an isolated $x_{0} \in K_{\varphi}^{c}$ are defined by

$$
C_{k}\left(\varphi, x_{0}\right)=H_{k}\left(\varphi^{c} \cap U,\left(\varphi^{c} \cap U\right) \backslash\left\{x_{0}\right\}\right), \text { for all } k \geq 0,
$$

where $U$ is a neighborhood of $x_{0}$ such that $K_{\varphi} \cap \varphi^{c} \cap U=\left\{x_{0}\right\}$. The excision property of the singular homology implies that this definition is independent of the particular choice of the neighborhood $U$.

Suppose that $\varphi \in C^{1}(X)$ satisfies the $C$-condition and $-\infty<\inf \varphi\left(K_{\varphi}\right)$. Let $c<\inf \varphi\left(K_{\varphi}\right)$. Then, the critical groups of $\varphi$ at infinity are defined by

$$
C_{k}(\varphi, \infty)=H_{k}\left(X, \varphi^{c}\right) \text { for all } k \geq 0 .
$$

The second deformation theorem (see, for example, Gasinski-Papageorgiou [20], p. $628)$ implies that this definition is independent of the choice of the level $c<\inf \varphi\left(K_{\varphi}\right)$.

If $K_{\varphi}$ is finite, then we define

$$
M(t, x)=\sum_{k \geq 0} \operatorname{rank} C_{k}(\varphi, x) t^{k}, \text { for all } x \in K_{\varphi}
$$

and

$$
P(t, \infty)=\sum_{k \geq 0} \operatorname{rank} C_{k}(\varphi, \infty) t^{k}, \text { for all } t \in \mathbb{R} .
$$

The Morse relation says that

$$
\sum_{x \in K_{\varphi}} M(t, x)=P(t, \infty)+(1+t) Q(t), t \in \mathbb{R},
$$

where $Q(t)=\sum_{k \geq 0} \beta_{k} t^{k}$ is a formal series with nonnegative integer coefficients. i.e.,

Throughout this work, by $\|\cdot\|$ we denote the norm of the Sobolev space $W_{0}^{1, p}(\Omega)$,

$$
\|u\|=\|D u\|_{p}
$$

(by virtue of the Poincaré inequality), where $\|\cdot\|_{p}$ stands for the norm in $L^{p}(\Omega)$ or $L^{p}\left(\Omega, \mathbb{R}^{N}\right)$. The notation $\|\cdot\|$ will be also used to denote the $\mathbb{R}^{N}$-norm. It will always be clear from the context, which norm we use. For $x \in \mathbb{R}$, we set

$$
x^{ \pm}=\max \{ \pm x, 0\} .
$$


Then for $u \in W_{0}^{1, p}(\Omega)$ we define $u^{ \pm}()=.u(.)^{ \pm}$. We know that $u^{ \pm} \in W_{0}^{1, p}(\Omega)$ and

$$
|u|=u^{+}+u^{-}, u=u^{+}-u^{-} .
$$

By $|\cdot|_{N}$ and $(., .)_{\mathbb{R}^{N}}$ we denote the Lebesgue measure on $\mathbb{R}^{N}$ and the inner product in $\mathbb{R}^{N}$, respectively. If $\theta: \Omega \times \mathbb{R} \rightarrow \mathbb{R}$ is a measurable function (for example, a Carathéodory function), then

$$
N_{\theta}(u)(.)=\theta(., u(.)) \text { for all } u \in W_{0}^{1, p}(\Omega) .
$$

Finally, we will use the symbol $\stackrel{w}{\longrightarrow}$ " to designate weak convergence.

3. Preliminary results. It what follows $h \in C^{1}(0, \infty)$ is such that

$$
\widehat{C} \leq \frac{t h^{\prime}(t)}{h(t)} \leq C_{0} \text { for all } t>0 \text { and some } \widehat{C}, C_{0}>0
$$

and

$$
C_{1} t^{p-1} \leq h(t) \leq C_{2}\left(t^{q_{0}-1}+t^{p-1}\right) \text { for all } t>0 \text { and some } C_{1}, C_{2}>0,
$$

with $1<q_{0} \leq p<\infty$. We introduce the following hypotheses on $a(z, y)$ :

$\left(\mathbf{H}_{0}\right) a(z, y)=a_{0}(z,\|y\|) y$, where the function $a_{0}: \bar{\Omega} \times(0, \infty) \rightarrow(0, \infty)$ satisfies

$$
\lim _{t \rightarrow 0^{+}} a_{0}(z, t) t=0 \text { for all } z \in \bar{\Omega}
$$

moreover

(i) $a \in C^{1}\left(\bar{\Omega} \times \mathbb{R}^{N} \backslash\{0\}, \mathbb{R}^{N}\right) \cap C\left(\bar{\Omega} \times \mathbb{R}^{N}, \mathbb{R}^{N}\right)$ and for every $K \subset$ $\mathbb{R}^{N} \backslash\{0\}$ compact, there exists $\beta=\beta(K) \in(0,1)$ such that $a \in$ $C^{0, \beta}\left(\bar{\Omega} \times K, \mathbb{R}^{N}\right)$

(ii) for all $(z, y) \in \bar{\Omega} \times \mathbb{R}^{N} \backslash\{0\}$ we have

$$
\frac{h(\|y\|)}{\|y\|}\|\xi\|^{2} \leq\left(\nabla_{y} a(z, y) \xi, \xi\right)_{\mathbb{R}^{N}} \text { for all } \xi \in \mathbb{R}^{N}
$$

(iii) for all $(z, y) \in \bar{\Omega} \times \mathbb{R}^{N} \backslash\{0\}$ we have

$$
\left\|\nabla_{y} a(z, y)\right\| \leq C_{3} \frac{h(\|y\|)}{\|y\|} \text { for some } C_{3}>0 ;
$$

(iv) the primitive $G(z, y)$ determined by

$$
\nabla_{y} G(z, y)=a(z, y) \forall(z, y) \in \bar{\Omega} \times \mathbb{R}^{N} \text { and } G(z, 0)=0 \forall z \in \bar{\Omega}
$$

satisfies

$$
k(z) \leq p G(z, y)-(a(z, y), y)_{\mathbb{R}^{N}} \text { for all } z \in \Omega, \text { all } y \in \mathbb{R}^{N},
$$

with $k \in L^{1}(\Omega)$;

(v) there exists $q \in(1, p)$ such that

$$
\lim _{y \rightarrow 0} \frac{G(z, y)}{\|y\|^{q}}=0 \text { uniformly for all } z \in \bar{\Omega}
$$

and if $G_{0}(z, t)=\int_{0}^{t} a_{0}(z, s) s d s$, for $t>0$, then for some $\tau \in(q, p)$, $t \rightarrow G_{0}\left(z, t^{\frac{1}{\tau}}\right)$ is convex. 
Remarks. Comparable conditions on $a($.$) can be found in Papageorgiou-$ Radulescu [32]. Let us present some straightforward but useful consequences of the above hypotheses. First, we show that for all $z \in \bar{\Omega}, a(z,$.$) is strictly monotone. To$ this end, let $y, y^{\prime} \in \mathbb{R}^{N}, y \neq y^{\prime}$. For all $z \in \bar{\Omega}$, we have

$$
\begin{aligned}
\left(a(z, y)-a\left(z, y^{\prime}\right), y-y^{\prime}\right)_{\mathbb{R}^{N}} & =\int_{0}^{1}\left(\frac{d}{d t} a\left(z, y^{\prime}+t\left(y-y^{\prime}\right), y-y^{\prime}\right)\right)_{\mathbb{R}^{N}} d t \\
& =\int_{0}^{1}\left(\nabla_{y} a\left(z, y^{\prime}+t\left(y-y^{\prime}\right)\right)\left(y-y^{\prime}\right), y-y^{\prime}\right)_{\mathbb{R}^{N}} d t \\
& \geq C_{1} \int_{0}^{1}\left\|y^{\prime}+t\left(y-y^{\prime}\right)\right\|^{p-2}\left\|y-y^{\prime}\right\|^{2} d t
\end{aligned}
$$

(see $\left(\mathbf{H}_{0}\right)($ ii $)$ and $\left.(3.1)\right)$, hence $a(z .$,$) is monotone. Hence for all z \in \bar{\Omega}, G(., 0)$ and $G_{0}(., 0)$ are both strictly convex. Also, we have

$$
a(z, y)=\int_{0}^{1} \frac{d}{d t} a(z, t y) d t=\int_{0}^{1} \nabla_{y} a(z, t y) y d t
$$

hence

$$
\begin{aligned}
\|a(z, y)\| & \leq \int_{0}^{1}\left\|\nabla_{y} a(z, t y)\right\|\|y\| d t \\
& \leq C_{4}\left(1+\|y\|^{p-1}\right) \text { for some } C_{4}>0, \text { for all }(z, y) \in \bar{\Omega} \times \mathbb{R}^{N}
\end{aligned}
$$

(see $\left(\mathbf{H}_{0}\right)($ iii $)$ and (3.1)). Moreover, we have

$$
\begin{aligned}
(a(z, y), y)_{\mathbb{R}^{N}} & =\int_{0}^{1}\left(\nabla_{y} a(z, t y) y, y\right)_{\mathbb{R}^{N}} d t \\
& \geq \frac{C_{1}}{p-1}\|y\|^{p} \text { for all }(z, y) \in \bar{\Omega} \times \mathbb{R}^{N}
\end{aligned}
$$

(see $\left(\mathbf{H}_{0}\right)(i i)$ and (3.1)). From (3.2) and (3.3) and since

$$
G(z, y)=\int_{0}^{1} \frac{d}{d t} G(z, t y) d t=\int_{0}^{1}(a(z, t y), y)_{\mathbb{R}^{N}} d t
$$

we obtain

(3.4) $\frac{C_{1}}{p(p-1)}\|y\|^{p} \leq G(z, y) \leq C_{5}\left(1+\|y\|^{p}\right)$ for some $C_{5}>0, \forall(z, y) \in \bar{\Omega} \times \mathbb{R}^{N}$.

EXAMPLES. The following maps satisfy hypotheses $\left(\mathbf{H}_{0}\right)$ :

(a) $a_{1}(z, y)=\theta(z)\|y\|^{p-2} y$ with $1<p<\infty, \theta \in C^{1}(\bar{\Omega}), \theta(z)>0 \forall z \in \bar{\Omega}$. 
This map corresponds to the weighted $p$ - Laplacian differential operator.

(b) $a_{1}(z, y)=\theta(z)\|y\|^{p-2} y+\widehat{\theta}(z)\|y\|^{q-2} y$ with $1<q<p<\infty, \theta, \widehat{\theta} \in C^{1}(\bar{\Omega})$, $\theta(z)>0, \widehat{\theta}(z)>0 \forall z \in \bar{\Omega}$.

This map corresponds to the weighted $(p, q)$ - Laplacian differential operator. Such operators are important in quantum physics, see, for example, Benci-FortunatoPisani [8]. Recently, there have been papers dealing with the $(p, q)$-Laplacian, see Cingolani-Degiovanni [12], Figueiredo [18] and Medeiros-Perera [30].

(c) $a_{3}(z, y)=\theta(z)\left(\|y\|^{p-2} y+c \frac{\|y\|^{q-2} y}{1+\|y\|^{p}}\right)$ with $1<q \leq p<\infty, \theta \in C^{1}(\bar{\Omega})$, $\theta(z)>0 \forall z \in \bar{\Omega}, c>0$.

(d) $a_{4}(y)=\left(1+\|y\|^{2}\right)^{\frac{p-2}{2}} y$, with $p>1$.

This correspond to the generalized $p$-mean curvature operator.

Let $A: W_{0}^{1, p}(\Omega) \rightarrow W_{0}^{1, p^{\prime}}(\Omega)=W_{0}^{1, p}(\Omega)^{*}\left(\frac{1}{p}+\frac{1}{p^{\prime}}=1\right)$ be the nonlinear map defined by

$$
\langle A(u), y\rangle=\int_{\Omega}((a(z, D u)), D y)_{\mathbb{R}^{N}} d z \text { for all } u, y \in W_{0}^{1, p}(\Omega) .
$$

From Papageorgiou-Rocha-Staicu [33] we have:

Proposition 1. If hypotheses $\left(\mathbf{H}_{0}\right)(i),($ ii $)$, (iii) hold, then the map $A$ : $W_{0}^{1, p}(\Omega) \rightarrow W^{-1, p^{\prime}}(\Omega)$ defined by $(3.5)$ is bounded, continuous, strictly monotone (hence maximal monotone too) and of type $(S)_{+}$, i.e., if $\left\{u_{n}\right\}_{n \geq 1} \subseteq W_{0}^{1, p}(\Omega)$ is such that $u_{n} \stackrel{w}{\longrightarrow} u$ in $W_{0}^{1, p}(\Omega)$ and

$$
\limsup _{n \rightarrow \infty}\left\langle A\left(u_{n}\right), u_{n}-u\right\rangle \leq 0
$$

then $u_{n} \rightarrow u$ in $W_{0}^{1, p}(\Omega)$ as $n \rightarrow \infty$.

Next let $f_{0}: \Omega \times \mathbb{R} \rightarrow \mathbb{R}$ be a Carathéodory function such that

$$
\left|f_{0}(z, x)\right| \leq \alpha(z)+c|x|^{r-1} \text { for a. a. } z \in \Omega \text {, all } x \in \mathbb{R}
$$

with $\alpha \in L^{\infty}(\Omega)_{+}, c>0$ and $1<r<p^{*}$. We set $F_{0}(z, x)=\int_{0}^{x} f_{0}(z, s) d s$ and consider the $C^{1}-$ functional $\varphi_{0}: W_{0}^{1, p}(\Omega) \rightarrow \mathbb{R}$ defined by

$$
\varphi_{0}(u)=\int_{\Omega} G(z, D u(z)) d z-\int_{\Omega} F_{0}(z, D u(z)) d z \text { for all } u \in W_{0}^{1, p}(\Omega) .
$$

The next proposition was proved by Aizicovici-Papageorgiou-Staicu [4]. Actually, the hypotheses on $a(z, x)$ in [4] are more restrictive (they exclude , for example, the $(p, q)$ - Laplacian differential operator. However, a careful reading of the proof of Proposition 2 in [4] reveals that it remains valid under the present more general hypotheses $\left(\mathbf{H}_{0}\right)$, provided we use instead the stronger regularity result of Lieberman ([28], p.320). So, we can state the following result.

Proposition 2. If $u_{0} \in W_{0}^{1, p}(\Omega)$ is a local $C_{0}^{1}(\bar{\Omega})-$ minimizer of $\varphi_{0}$ (i.e., there exists $r_{0}>0$ such that $\varphi_{0}\left(u_{0}\right) \leq \varphi_{0}\left(u_{0}+h\right)$ for all $h \in C_{0}^{1}(\bar{\Omega})$ with $\left.\|h\|_{C_{0}^{1}(\bar{\Omega})} \leq r_{0}\right)$ 
then $u_{0} \in C_{0}^{1, \beta}(\bar{\Omega})$ with $\beta \in(0,1)$ and it is a $W_{0}^{1, p}(\Omega)-$ minimizer of $\varphi_{0}$ (i.e., there exists $r_{1}>0$ such that $\varphi_{0}\left(u_{0}\right) \leq \varphi_{0}\left(u_{0}+h\right)$ for all $h \in W_{0}^{1, p}(\Omega)$ with $\|h\|_{W_{0}^{1, p}(\Omega)} \leq$ $\left.r_{1}\right)$.

REMARKS. This result was first proved for $G(z, y)=G(y)=\frac{1}{2}\|y\|^{2}$ (corresponding to the Laplace differential operator) by Brezis-Nirenberg [10] and was extended to the case $G(z, y)=G(y)=\frac{1}{p}\|y\|^{p}, 1<p<\infty$ (corresponding to the $p$-Laplace differential operator) by Garcia Azorero-Manfredi-Peral Alonso [19]. See also GuoZhang [21] where $2 \leq p<\infty$.

Now we consider the following auxiliary Dirichlet problem

$$
-\operatorname{div} a(z, D u(z))=\widehat{f}(z, u(z)) \text { in } \Omega,\left.u\right|_{\partial \Omega}=0 .
$$

We are interested in the uniqueness of the nontrivial positive and negative solutions of (3.6), when they exist. To this end, we impose the following conditions of the reaction $\widehat{f}(z, x)$ :

$\left(\mathbf{H}_{1}\right) \widehat{f}: \Omega \times \mathbb{R}^{N} \rightarrow \mathbb{R}$ is a Carathéodory function such that $\widehat{f}(z, 0)=0$ a. e. in $\Omega$ and

(i) $|\widehat{f}(z, x)| \leq \alpha(z)+C|z|^{r-1}$ for a.a. $z \in \Omega$, all $x \in \mathbb{R}$, with $\alpha \in L^{\infty}(\Omega)_{+}$, $C>0$ and $1<r<p^{*}$;

(ii) for a.a. $z \in \Omega, x \rightarrow \frac{\widehat{f}(z, x)}{x^{\tau-1}}$ is strictly decreasing on $(0, \infty)$ and $x \rightarrow$ $\frac{\widehat{f}(z, x)}{|x|^{\tau-2} x}$ is strictly increasing on $(-\infty, 0)$, where $\tau$ is as in $\left(\mathbf{H}_{0}\right)(v)$, while $\widehat{f}(z, x) x \geq 0$ for a.a. $z \in \Omega$, all $x \in \mathbb{R}$.

In what follows, in addition to the Sobolev space $W_{0}^{1, p}(\Omega)$, we also use the Banach space

$$
C_{0}^{1}(\bar{\Omega})=\left\{u \in C^{1}(\bar{\Omega}):\left.u\right|_{\partial \Omega}=0\right\} .
$$

This is an ordered Banach space with positive cone

$$
C_{+}=\left\{u \in C_{0}^{1}(\bar{\Omega}): u(z) \geq 0 \text { for all } z \in \bar{\Omega}\right\} .
$$

This cone has a nonempty interior, given by

$$
\text { int } C_{+}=\left\{u \in C_{+}: u(z)>0 \text { for all } z \in \Omega, \frac{\partial u}{\partial n}(z)<0 \text { for all } z \in \partial \Omega\right\},
$$

where by $n($.$) we denote the outward unit normal on \partial \Omega$.

Proposition 3. If hypotheses $\left(\mathbf{H}_{0}\right)$ and $\left(\mathbf{H}_{1}\right)$ hold, then problem (3.6) has at most one nontrivial positive solution belonging to int $C_{+}$and at most one nontrivial negative solution belonging to - int $C_{+}$.

Proof. We show the uniqueness of the nontrivial positive solution (when it exists), the proof of the nontrivial negative solution (when it exists) being similar.

So, let $u \in W_{0}^{1, p}(\Omega)$ be a nontrivial positive solution of (3.6). Then

$$
-\operatorname{div} a(z, D u(z))=\widehat{f}(z, u(z)) \text { a.e. in } \Omega,\left.u\right|_{\partial \Omega}=0 \text {. }
$$


From Ladyzhenskaya-Uraltseva ([26], p.286), we have that $u \in L^{\infty}(\Omega)$. Then, invoking the regularity result of Lieberman ([28], p.320), we infer that $u \in C_{+} \backslash\{0\}$. Moreover, by virtue of hypothesis $\left(\mathbf{H}_{1}\right)(i i)$, we have

$$
\operatorname{div} a(z, D u(z)) \leq 0 \text { a.e. in } \Omega \text {, }
$$

hence

$$
u \in \operatorname{int} C_{+}
$$

(see Cuesta-Takac [14], Theorem 2.1). The result of [14] is formulated under a little more restrictive hypotheses on $a(z, y)$, but it remains valid under the present more general conditions, thanks to the regularity results of Lieberman [28].

Let $\gamma: L^{1}(\Omega) \rightarrow \overline{\mathbb{R}}=\mathbb{R} \cup\{+\infty\}$ be the integral functional defined by

$$
\gamma(u)= \begin{cases}\int_{\Omega} G\left(z, D u^{\frac{1}{\tau}}\right) d z & \text { if } u \geq 0, u^{\frac{1}{\tau}} \in W_{0}^{1, p}(\Omega) \\ +\infty & \text { otherwise }\end{cases}
$$

Let $u_{1}, u_{2} \in d o m \gamma$ and set

$$
y=\left[t u_{1}+(1-t) u_{2}\right]^{\frac{1}{\tau}} \text { for } t \in(0,1) \text { and } v_{1}=u_{1}^{\frac{1}{\tau}}, v_{2}=u_{2}^{\frac{1}{\tau}} .
$$

As in Diaz-Saa [15] (see Lemma 1; see also Benguria-Brezis-Lieb ([9], Lemma 4), using Holder's inequality, we have

$$
\|D y(z)\| \leq\left(t\left\|D v_{1}(z)\right\|^{\tau}+(1-t)\left\|D v_{2}(z)\right\|^{\tau}\right)^{\frac{1}{\tau}} \text { for all } z \in \bar{\Omega} .
$$

Since for all $z \in \bar{\Omega}, G_{0}(z,$.$) is increasing, we have$

$$
\begin{aligned}
G_{0}(z,\|D y(z)\|) & \leq G_{0}\left(z,\left(t\left\|D v_{1}(z)\right\|^{\tau}+(1-t)\left\|D v_{2}(z)\right\|^{\tau}\right)^{\frac{1}{\tau}}\right) \\
& \leq t G_{0}\left(z,\left\|D v_{1}(z)\right\|\right)+(1-t) G_{0}\left(z,\left\|D v_{2}(z)\right\|\right) \text { for all } z \in \bar{\Omega}
\end{aligned}
$$

$\left(\right.$ see $\left.\left(\mathbf{H}_{0}\right)(v)\right)$. Note that $G(z, y)=G_{0}(z,\|y\|)$ for all $(z, y) \in \bar{\Omega} \times \mathbb{R}^{N}$. Hence we arrive at

$$
G(z, D y(z)) \leq t G\left(z, D u_{1}(z)^{\frac{1}{\tau}}\right)+(1-t) G\left(z, D u_{2}(z)^{\frac{1}{\tau}}\right) \text { for all } z \in \bar{\Omega},
$$

therefore $\gamma($.$) is convex.$

Moreover, using Fatou's lemma, we show that $\gamma($.$) is lower semicontinuous. Fi-$ nally, $\gamma($.$) is not identically +\infty$ (i.e., $\operatorname{dom} \gamma \neq \varnothing$ ).

Let $u$ be a nontrivial positive solution of (3.6). From the first part of the proof we know that $u \in$ int $C_{+}$. Then $u^{\tau} \geq 0,\left(u^{\tau}\right)^{\frac{1}{\tau}}=u \in W_{0}^{1, p}(\Omega)$ and so, $u^{\tau} \in$ dom $\gamma$. Let $h \in C_{0}^{1}(\bar{\Omega})$ and $r>0$ small. Then $u^{\tau}+r h \in C_{+}$and so the Gateaux derivative of $\gamma($.$) at u^{\tau}$ in the direction $h$ exists. Moreover, using the chain rule, we have

$$
\gamma^{\prime}\left(u^{\tau}\right)(h)=\frac{1}{\tau} \int_{\Omega} \frac{-\operatorname{div} a(z, D u)}{u^{\tau-1}} h d z .
$$


Let $v$ be another nontrivial positive solution of (3.6). As above, we have $v \in$ int $C_{+}$ and (3.7) holds with $u$ replaced by $v$. The convexity of $\gamma($.$) implies that y \rightarrow \gamma^{\prime}(y)$ is monotone. Hence

$$
\begin{aligned}
0 & \leq \int_{\Omega}\left(\frac{-\operatorname{div} a(z, D u)}{u^{\tau-1}}+\frac{\operatorname{div} a(z, D v)}{v^{\tau-1}}\right)(u-v) d z \\
& =\int_{\Omega}\left(\frac{\widehat{f}(z, u)}{u^{\tau-1}}-\frac{\widehat{f}(z, v)}{v^{\tau-1}}\right)(u-v) d z
\end{aligned}
$$

Since $x \rightarrow \frac{\widehat{f}(z, x)}{x^{\tau-1}}$ is strictly decreasing on $(0, \infty)$ (see $\left.\left(\mathbf{H}_{1}\right)(i i)\right)$, from (3.6) we infer that $u=v$. This proves the uniqueness of the nontrivial positive solution, when it exists. Similarly, for the nontrivial negative solution.

4. Constant sign solutions. In this section we produce four constant sign smooth solutions of $\left(P_{\lambda}\right)$ for all suitably small $\lambda>0$. The hypotheses on the reaction $f(z, x, \lambda)$ are the following:

$\left(\mathbf{H}_{2}\right): f: \Omega \times \mathbb{R} \times(0, \infty) \rightarrow \mathbb{R}$ is a function such that for all $\lambda>0,(z, x) \rightarrow f(z, x, \lambda)$ is a Carathéodory function, $f(z, 0, \lambda)=0$ a.e. in $\Omega$ and

(i) for every $\rho>0$ and $\lambda>0$, there exists $\alpha_{\rho}(., \lambda) \in L^{\infty}(\Omega)_{+}$such that

$$
|f(z, x, \lambda)| \leq \alpha_{\rho}(z, \lambda) \text { for a.a. } z \in \Omega \text {, all } x \in \mathbb{R} \text { with }|x| \leq \rho ;
$$

(ii) if $F(z, x, \lambda)=\int_{0}^{x} f(z, s, \lambda) d s$ then

$$
\lim _{x \rightarrow \pm \infty} \frac{F(z, x, \lambda)}{|x|^{p}}=+\infty \text { uniformly for a.a. } z \in \Omega
$$

and there exist $r \in\left(p, p^{*}\right)$ and $\widehat{\eta}_{\infty}, \eta_{\infty} \in L^{\infty}(\Omega)$ such that for every $\lambda>0$, we have

$$
\widehat{\eta}_{\infty}(z) \leq \liminf _{x \rightarrow \pm \infty} \frac{f(z, x, \lambda)}{|x|^{r-2} x} \leq \limsup _{x \rightarrow \pm \infty} \frac{f(z, x, \lambda)}{|x|^{r-2} x} \leq \eta_{\infty}(z)
$$

uniformly for a.a. $z \in \Omega$;

(iii) for every $\lambda>0$, there exists $\tau_{0}=\tau_{0}(\lambda) \in\left((r-p) \max \left\{1, \frac{N}{p}\right\}, p^{*}\right)$ and $\beta_{0}=\beta_{0}(\lambda)>0$ such that

$$
\beta_{0} \leq \liminf _{x \rightarrow \pm \infty} \frac{f(z, x, \lambda) x-p F(z, x, \lambda)}{|x|^{\tau_{0}}} \text { uniformly for a.a. } z \in \Omega ;
$$

(iv) if $q \in(1, p)$ is as in hypothesis $\left(\mathbf{H}_{0}\right)(v)$, then for all $\lambda>0$ we have

$$
\begin{aligned}
\widehat{C}_{0}|x|^{q} & \leq f(z, x, \lambda) x \text { for a.a. } z \in \Omega, \text { all } x \in \mathbb{R}, \\
\text { with } \widehat{C}_{0} & =\widehat{C}_{0}(\lambda)>0,
\end{aligned}
$$

there exists $\delta_{0}=\delta_{0}(\lambda)>0$ such that

$$
\begin{aligned}
& 0<f(z, x, \lambda) x \leq q F(z, x, \lambda) \text { for a.a. } z \in \Omega \text {, all }|x| \leq \delta_{0}, \\
& \text { ess inf } F\left(., \delta_{0}, \lambda\right)>0,
\end{aligned}
$$


and there exists $\eta_{0}(., \lambda) \in L^{\infty}(\Omega)_{+}$with $\left\|\eta_{0}(., \lambda)\right\|_{\infty} \rightarrow 0$ as $\lambda \rightarrow 0^{+}$ and

$$
\limsup _{x \rightarrow 0} \frac{F(z, x, \lambda)}{|x|^{q}} \leq \eta_{0}(z, \lambda) \text { uniformly for a.a. } z \in \Omega .
$$

Remarks. Hypothesis $\left(\mathbf{H}_{2}\right)(i i)$ implies that the primitive $F(z, ., \lambda)(\lambda>0)$ is $p$-superlinear near $\pm \infty$. However, note that we do not use the usual in such cases AR-condition. We recall that the AR-condition states that for every $\lambda>0$, there exists $\mu=\mu(\lambda)>p$ and $M=M(\lambda)>0$ such that

$$
\begin{aligned}
& 0<\mu F(z, x, \lambda) \leq f(z, x, \lambda) x \text { for a.a. } z \in \Omega \text {, all }|x| \geq M \text {, and } \\
& \text { ess inf } F(., M, \lambda)>0 . \\
& \quad \Omega
\end{aligned}
$$

From (4.1) we obtain the weaker condition

(4.2) $\widehat{C}_{1}|x|^{\mu} \leq F(z, x, \lambda)$ for a.a. $z \in \Omega$, all $x \in \mathbb{R}$ with $|x| \geq M$ and some $\widehat{C}_{1}>0$.

From (4.2) we infer the much weaker condition

$$
\lim _{x \rightarrow \pm \infty} \frac{F(z, x, \lambda)}{|x|^{p}}=+\infty \text { uniformly for a.a. } z \in \Omega .
$$

We replace the AR-condition (see (4.1)) by hypothesis $\left(\mathbf{H}_{2}\right)($ iii) which is weaker. Indeed, the AR-condition implies $\left(\mathbf{H}_{2}\right)$ (iii). To see this, note that we may assume that $(r-p) \max \left\{1, \frac{N}{p}\right\}<\mu$ and then for all $\lambda>0$, we have

$$
\begin{aligned}
\frac{f(z, x, \lambda) x-p F(z, x, \lambda)}{|x|^{\mu}} & =\frac{f(z, x, \lambda) x-\mu F(z, x, \lambda)}{|x|^{\mu}}+\frac{(\mu-p) F(z, x, \lambda)}{|x|^{\mu}} \\
& \geq(\mu-p) \widehat{C}_{1} \text { for a.a. } z \in \Omega, \text { all }|x| \geq M,
\end{aligned}
$$

by (4.1) and (4.2), and so $\left(\mathbf{H}_{2}\right)$ (iii) holds. This alternative "superlinearity" condition incorporates in our setting "superlinear" nonlinearities with "slower" growth condition at $\pm \infty$ which fail to satisfy the AR-condition. Similar conditions can be found in the works of Costa-Magalhães [13] and Fei [17].

EXAMPLES. The following functions satisfy hypotheses $\left(\mathbf{H}_{2}\right)$ (for the sake of simplicity we drop the $z$ - dependence):

$$
\begin{aligned}
& f_{1}(x, \lambda)=\lambda|x|^{q-2} x+|x|^{r-2} x \text { with } 1<q<p<r<p^{*} ; \\
& f_{2}(x, \lambda)=\lambda\left(|x|^{q-2} x+|x|^{r-2} x\right) \text { with } 1<q<p<r<p^{*} ; \\
& f_{3}(x, \lambda)=\lambda|x|^{q-2} x+|x|^{p-2} x \ln (1+|x|) \text { with } 1<q<p<\infty ; \\
& f_{4}(x, \lambda)= \begin{cases}|x|^{r-2} x-\xi(\lambda) & \text { if } \quad x<-\rho(\lambda) \\
|x|^{q-2} x & \text { if }-\rho(\lambda) \leq x \leq \rho(\lambda) \\
|x|^{r-1}+\xi(\lambda) & \text { if } \rho(\lambda)<x\end{cases} \\
& \text { with } \xi(\lambda)=\left(1-\rho(\lambda)^{r-q}\right) \rho(\lambda)^{q-1}, 1<q<p<r<p^{*} \text { and } \\
& \rho(\lambda) \in(0,1), \rho(\lambda) \rightarrow 0^{+} \text {as } \lambda \rightarrow 0^{+} .
\end{aligned}
$$


Note that $f_{3}(., \lambda)$ does not satisfy the AR-condition.

Let

$$
f_{ \pm}(z, x, \lambda)=f\left(z, \pm x^{ \pm}, \lambda\right) .
$$

These are Carathéodory functions. We set

$$
F_{ \pm}(z, x, \lambda)=\int_{0}^{x} f_{ \pm}(z, s, \lambda) d s
$$

and consider the $C^{1}$-functionals $\varphi_{ \pm}^{\lambda}: W_{0}^{1, p}(\Omega) \rightarrow \mathbb{R}$ defined by

$$
\varphi_{ \pm}^{\lambda}(u)=\int_{\Omega} G(z, D u(z)) d z-\int_{\Omega} F_{ \pm}(z, u(z), \lambda) d z \text { for all } u \in W_{0}^{1, p}(\Omega) .
$$

Also $\varphi_{\lambda}: W_{0}^{1, p}(\Omega) \rightarrow \mathbb{R}$ is the $C^{1}-$ energy functional of problem $\left(P_{\lambda}\right)$ defined by

$$
\varphi_{\lambda}(u)=\int_{\Omega} G(z, D u(z)) d z-\int_{\Omega} F(z, u(z), \lambda) d z \text { for all } u \in W_{0}^{1, p}(\Omega) .
$$

Proposition 4. If hypotheses $\left(\mathbf{H}_{0}\right)$ and $\left(\mathbf{H}_{2}\right)$ hold and $\lambda>0$, then the functionals $\varphi_{ \pm}^{\lambda}: W_{0}^{1, p}(\Omega) \rightarrow \mathbb{R}$ satisfy the $C$-condition.

Proof. We complete the proof for $\varphi_{+}^{\lambda}$. Let $\left\{u_{n}\right\}_{n \geq 1} \subset W_{0}^{1, p}(\Omega)$ be a sequence such that

$$
\left|\varphi_{+}^{\lambda}\left(u_{n}\right)\right| \leq M_{1} \text { for some } M_{1}>0, \text { all } n \geq 1
$$

and

$$
\left(1+\left\|u_{n}\right\|\right)\left(\varphi_{+}^{\lambda}\right)^{\prime}\left(u_{n}\right) \rightarrow 0 \text { in } W^{-1, p^{\prime}}(\Omega) \text { as } n \rightarrow \infty .
$$

From (4.4) we have

$$
\left|\left\langle\left(\varphi_{+}^{\lambda}\right)^{\prime}\left(u_{n}\right), v\right\rangle\right| \leq \frac{\varepsilon_{n}\|v\|}{1+\left\|u_{n}\right\|} \text { for all } v \in W_{0}^{1, p}(\Omega) \text { with } \varepsilon_{n} \rightarrow 0^{+},
$$

hence

$$
\left|\left\langle A\left(u_{n}\right), v\right\rangle-\int_{\Omega} f_{+}\left(z, u_{n}, \lambda\right) v d z\right| \leq \frac{\varepsilon_{n}\|v\|}{1+\left\|u_{n}\right\|} \text { for all } v \in W_{0}^{1, p}(\Omega), \text { all } n \geq 1 .
$$

In (4.5) we choose $v=-u_{n}^{-} \in W_{0}^{1, p}(\Omega)$. Then

$$
\int_{\Omega}\left(a\left(z,-D u_{n}^{-}\right),-D u_{n}^{-}\right)_{\mathbb{R}^{N}} d z \leq \varepsilon_{n} \text { for all } n \geq 1,
$$

hence

$$
\frac{C_{1}}{p-1}\left\|D u_{n}^{-}\right\|_{p}^{p} \leq \varepsilon_{n} \text { for all } n \geq 1(\text { see }(3.3))
$$

therefore

$$
u_{n}^{-} \rightarrow 0 \text { in } W_{0}^{1, p}(\Omega) \text { as } n \rightarrow \infty .
$$


Next in (4.5) we choose $v=u_{n}^{+} \in W_{0}^{1, p}(\Omega)$. Then

$$
-\int_{\Omega}\left(a\left(z, D u_{n}^{+}\right), D u_{n}^{+}\right)_{\mathbb{R}^{N}} d z+\int_{\Omega} f\left(z, u_{n}^{+}, \lambda\right) u_{n}^{+} d z \leq \varepsilon_{n} \text { for all } n \geq 1 .
$$

On the other hand from (4.3) and (4.6), we have

$$
\int_{\Omega} p G\left(z, D u_{n}^{+}\right) d z-\int_{\Omega} p F\left(z, u_{n}^{+}, \lambda\right) d z \leq M_{2} \text { for some } M_{2}>0, \text { all } n \geq 1 .
$$

Adding (4.7) and (4.8), we obtain

$$
\begin{aligned}
& \int_{\Omega}\left[p G\left(z, D u_{n}^{+}\right)-\left(a\left(z, D u_{n}^{+}\right), D u_{n}^{+}\right)_{\mathbb{R}^{N}}\right] d z+\int_{\Omega}\left[f\left(z, u_{n}^{+}, \lambda\right) u_{n}^{+}-p F\left(z, u_{n}^{+}, \lambda\right)\right] d z \\
& \leq M_{3} \text { for some } M_{3}>0, \text { all } n \geq 1,
\end{aligned}
$$

hence

$$
\int_{\Omega}\left[f\left(z, u_{n}^{+}, \lambda\right) u_{n}^{+}-p F\left(z, u_{n}^{+}, \lambda\right)\right] d z \leq M_{4} \text { for some } M_{4}>0, \text { all } n \geq 1
$$

(see $\left.\left(\mathbf{H}_{0}\right)(i v)\right)$. Hypotheses $\left(\mathbf{H}_{2}\right)(i)$, (iii) imply that we can find $\widehat{\beta}_{0} \in\left(0, \beta_{0}\right)$ and $C_{6}>0$ such that

$$
\widehat{\beta}_{0}|x|^{\tau_{0}}-C_{6} \leq f(z, x, \lambda) x-p F(z, x, \lambda) \text { for a.a. } z \in \Omega \text {, all } x \in \mathbb{R} .
$$

Using (4.10) in (4.9) we see that

$$
\left\{u_{n}^{+}\right\}_{n \geq 1} \subset L^{\tau_{0}}(\Omega) \text { is bounded. }
$$

First we suppose that $N \neq p$. It is clear from hypothesis $\left(\mathbf{H}_{2}\right)($ iii $)$ that without any loss of generality, we may assume that $1<\tau_{0} \leq r<p^{*}$. So, we can find $t \in[0,1)$ such that

$$
\frac{1}{r}=\frac{1-t}{\tau_{0}}+\frac{t}{p^{*}}
$$

Invoking the interpolation inequality (see, for example, Gasinski-Papageorgiou ([20], p.905), we have

$$
\left\|u_{n}^{+}\right\|_{r} \leq\left\|u_{n}^{+}\right\|_{\tau_{0}}^{1-t}\left\|u_{n}^{+}\right\|_{p *}^{t}
$$

hence

$$
\left\|u_{n}^{+}\right\|_{r}^{r} \leq M_{5}\left\|u_{n}^{+}\right\|^{t r} \text { for some } M_{5}>0, \text { all } n \geq 1
$$

(see (4.11)). It is clear that hypotheses $\left(\mathbf{H}_{2}\right)$ imply

(4.13) $0 \leq f(z, x, \lambda) x \leq \widehat{a}(z, \lambda)+\widehat{C}(\lambda)|x|^{r}$ for a.a. $z \in \Omega$, all $x \in \mathbb{R}$, all $\lambda>0$, with $\widehat{a}(., \lambda) \in L^{\infty}(\Omega)_{+}, \widehat{C}(\lambda)>0$. In $(4.5)$, we choose $v=u_{n}^{+} \in W_{0}^{1, p}(\Omega)$. Then

$$
\int_{\Omega}\left(a\left(z, D u_{n}^{+}\right), D u_{n}^{+}\right)_{\mathbb{R}^{N}} d z-\int_{\Omega} f\left(z, u_{n}^{+}, \lambda\right) u_{n}^{+} d z \leq \varepsilon_{n} \text { for all } n \geq 1,
$$


hence

$$
\frac{C_{1}}{p-1}\left\|D u_{n}^{+}\right\|_{p}^{p}-\int_{\Omega} f\left(z, u_{n}^{+}, \lambda\right) u_{n}^{+} d z \leq \varepsilon_{n} \text { for all } n \geq 1(\text { see }(3.3))
$$

therefore

$$
\left\|u_{n}^{+}\right\|^{p} \leq C_{7}\left(1+\left\|u_{n}^{+}\right\|_{r}^{r}\right) \text { for all } n \geq 1 \text { and some } C_{7}=C_{7}(\lambda)>0
$$

(see (4.13)), and by using (4.12) we conclude that

$$
\left\|u_{n}^{+}\right\|^{p} \leq C_{8}\left(1+\left\|u_{n}^{+}\right\|^{t r}\right) \text { for all } n \geq 1 \text { and some } C_{8}=C_{8}(\lambda)>0 .
$$

The condition on $\tau_{0}$ (see $\left.\left(\mathbf{H}_{2}\right)(i i i)\right)$ implies that $t r<p$, and so, from (4.14) it follows that

$$
\left\{u_{n}^{+}\right\}_{n \geq 1} \subset W_{0}^{1, p}(\Omega) \text { is bounded, }
$$

hence

$$
\left\{u_{n}\right\}_{n \geq 1} \subset W_{0}^{1, p}(\Omega) \text { is bounded (see (4.6)). }
$$

Now assume that $N=p$. In this case, by definition, $p^{*}=+\infty$, while the Sobolev embedding theorem implies that $W_{0}^{1, p}(\Omega)$ is embedded compactly in $L^{\theta}(\Omega)$ for all $\theta \in[1, \infty)$. So, in order to employ the previous argument, we replace $p^{*}$ by $\theta>r \geq$ $\tau_{0}>1$ and choose $t \in[0,1)$ such that

$$
\frac{1}{r}=\frac{1-t}{\tau_{0}}+\frac{t}{\theta}
$$

which implies

$$
\operatorname{tr}=\frac{\theta\left(r-\tau_{0}\right)}{\theta-\tau_{0}}
$$

Note that $\frac{\theta\left(r-\tau_{0}\right)}{\theta-\tau_{0}} \rightarrow r-\tau_{0}$ as $\theta \rightarrow+\infty=p^{*}$. Also, since $N=p$, from $\left(\mathbf{H}_{2}\right)(i i i)$, we have $r-\tau_{0}<p$. Therefore for $\theta>r$ large, we will have $t r<p$ (see (4.16)). With such a $\theta>r$ replacing $p^{*}$ in the previous argument, again we arrive at (4.15)).

Because of (4.15), we may assume that

$$
u_{n} \stackrel{w}{\longrightarrow} u \text { in } W_{0}^{1, p}(\Omega) \text { and } u_{n} \rightarrow u \text { in } L^{r}(\Omega) \text { as } n \rightarrow \infty .
$$

In (4.5) we choose $v=u_{n}-u$, pass to the limit as $n \rightarrow \infty$ and use (4.17). Then

$$
\lim _{n \rightarrow \infty}\left\langle A\left(u_{n}\right), u_{n}-u\right\rangle=0
$$

hence

$$
u_{n} \rightarrow u \text { in } W_{0}^{1, p}(\Omega) \text { as } n \rightarrow \infty
$$

(see Proposition 1) and we conclude that $\varphi_{+}^{\lambda}$ satisfies the C-condition. The proof for $\varphi_{-}^{\lambda}$ is similar.

With minor straightforward modifications in the previous proof, we also establish the following result. 
Proposition 5. If hypotheses $\left(\mathbf{H}_{0}\right)$ and $\left(\mathbf{H}_{2}\right)$ hold and $\lambda>0$, then the functional $\varphi_{\lambda}$ satisfies the $C$-condition.

The next proposition is important in the study of the mountain pass geometry.

Proposition 6. If hypotheses $\left(\mathbf{H}_{0}\right)$ and $\left(\mathbf{H}_{2}\right)$ hold, then there exist $\lambda_{ \pm}^{*}>0$, such that for every $\lambda \in\left(0, \lambda_{ \pm}^{*}\right)$ we can find $\rho_{\lambda}^{ \pm}>0$ for which we have

$$
\inf \left\{\varphi_{ \pm}^{\lambda}(u):\|u\|=\rho_{\lambda}^{ \pm}\right\}:=\eta_{ \pm}^{\lambda}>0 .
$$

Proof. Hypotheses $\left(\mathbf{H}_{2}\right)(i i),(i v)$ imply that we can find $C_{9}(\lambda)>0$ with $C_{9}(\lambda) \rightarrow$ $0^{+}$as $\lambda \rightarrow 0^{+}$and $C_{10}>0$, such that

$$
F(z, x, \lambda) \leq C_{9}(\lambda)|x|^{q}+C_{10}|x|^{r} \text { for a.a. } z \in \Omega \text {, all } x \in \mathbb{R} .
$$

For every $u \in W_{0}^{1, p}(\Omega)$, we have

$$
\begin{aligned}
\varphi_{+}^{\lambda}(u) & =\int_{\Omega} G(z, D u(z)) d z-\int_{\Omega} F_{+}(z, u(z), \lambda) d z \\
& \geq \frac{C_{1}}{p(p-1)}\|D u\|_{p}^{p}-\int_{\Omega} F\left(z, u^{+}, \lambda\right) d z(\text { see }(3.4)) \\
& \geq \frac{C_{1}}{p(p-1)}\|D u\|_{p}^{p}-C_{11}(\lambda)\|u\|^{q}-C_{12}^{q}\|u\|^{r} \text { (see (4.18)) } \\
& \text { for some } C_{11}(\lambda)>0 \text { with } C_{11}(\lambda) \rightarrow 0^{+} \text {as } \lambda \rightarrow 0^{+}, \text {and } C_{12}>0
\end{aligned}
$$

hence

$$
\varphi_{+}^{\lambda}(u) \geq\left[\frac{C_{1}}{p(p-1)}-C_{11}(\lambda)\|u\|^{q-p}-C_{12}\|u\|^{r-p}\right]\|u\|^{p} .
$$

We consider the function

$$
\xi_{\lambda}(t)=C_{11}(\lambda) t^{q-p}+C_{12} t^{r-p} \text { for all } t>0 .
$$

Evidently $\xi_{\lambda} \in C^{1}(0,+\infty)$ and since $q<p<r$, we have

$$
\xi_{\lambda}(t) \rightarrow+\infty \text { as } t \rightarrow 0^{+} \text {and } t \rightarrow+\infty \text {. }
$$

Therefore, we can find $t_{0} \in(0,+\infty)$ such that

$$
\xi_{\lambda}\left(t_{0}\right)=\inf \left\{\xi_{\lambda}(t): t>0\right\}
$$

hence

$$
\xi_{\lambda}^{\prime}\left(t_{0}\right)=C_{11}(\lambda)(q-p) t_{0}^{q-p-1}+C_{12}(r-p) t_{0}^{r-p-1}=0
$$

and we get

$$
t_{0}=t_{0}(\lambda)=\left[\frac{C_{11}(\lambda)(p-q)}{C_{12}(r-p)}\right]^{\frac{1}{r-q}} .
$$

We consider now $\xi_{\lambda}\left(t_{0}\right)$. By (4.20) and (4.21) and since $C_{11}(\lambda) \rightarrow 0^{+}$as $\lambda \rightarrow 0^{+}$, it follows that $\xi_{\lambda}\left(t_{0}\right) \rightarrow 0^{+}$as $\lambda \rightarrow 0^{+}$. Therefore, we can find $\lambda_{+}^{*}>0$ such that

$$
\xi_{\lambda}\left(t_{0}\right)<\frac{C_{1}}{p(p-1)} \text { for all } \lambda \in\left(0, \lambda_{+}^{*}\right)
$$


hence

$$
\varphi_{+}^{\lambda}(u) \geq \eta_{+}^{\lambda}>0 \text { for all } u \in W_{0}^{1, p}(\Omega) \text { with }\|u\|=\rho_{\lambda}^{+}:=t_{0}(\lambda)
$$

(see (4.19)). Similarly for $\varphi_{-}^{\lambda}$.

Now we are ready to produce nontrivial constant sign smooth solutions for problem $\left(P_{\lambda}\right)$. In what follows

$$
\lambda^{*}=\min \left\{\lambda_{+}^{*}, \lambda_{-}^{*}\right\}
$$

Proposition 7. If hypotheses $\left(\mathbf{H}_{0}\right)$ and $\left(\mathbf{H}_{2}\right)$ hold, then:

(a) for every $\lambda \in\left(0, \lambda_{+}^{*}\right)$, problem $\left(P_{\lambda}\right)$ has at least two nontrivial positive solutions $u_{0}, \widehat{u} \in$ int $C_{+}$, with $\widehat{u}$ a local minimizer of $\varphi_{\lambda}$;

(b) for every $\lambda \in\left(0, \lambda_{-}^{*}\right)$, problem $\left(P_{\lambda}\right)$ has at least two nontrivial negative solutions $v_{0}, \widehat{v} \in-$ int $C_{+}$, with $\widehat{v}$ a local minimizer of $\varphi_{\lambda}$;

(c) for every $\lambda \in\left(0, \lambda^{*}\right)$ problem $\left(P_{\lambda}\right)$ has at least four nontrivial smooth solutions of constant sign $u_{0}, \widehat{u} \in$ int $C_{+}, v_{0}, \widehat{v} \in-$ int $C_{+}$, with $\widehat{u}, \widehat{v}$ local minimizers of $\varphi_{\lambda}$;

Proof. (a) Let $\lambda \in\left(0, \lambda_{+}^{*}\right)$. First we show that

$$
\inf \left\{\varphi_{+}^{\lambda}(u):\|u\| \leq \rho_{\lambda}^{+}\right\}<0 .
$$

To this end, note that by virtue of hypothesis $\left(\mathbf{H}_{0}\right)(v)$, given $\varepsilon>0$, we can find $\delta=\delta(\varepsilon)>0$ such that

$$
G(z, y) \leq \varepsilon\|y\|^{q} \text { for all } z \in \bar{\Omega}, \text { all }\|y\| \leq \delta .
$$

Let $\widetilde{u} \in$ int $C_{+}$and choose $t \in(0,1)$ small, such that

$$
\|t \widetilde{u}(z)\| \leq \rho_{\lambda}^{+} \text {and }\|D(t \widetilde{u})(z)\| \leq \delta \text { for all } z \in \bar{\Omega}
$$

Then we have

$$
\begin{aligned}
& \varphi_{+}^{\lambda}(t \widetilde{u})=\int_{\Omega} G(z, D(t \widetilde{u})) d z-\int_{\Omega} F_{+}(z, t \widetilde{u}, \lambda) d z \\
& \leq \varepsilon t^{q}\|D \widetilde{u}\|_{q}^{q}-\widehat{C}_{0} t^{q}\|\widetilde{u}\|_{q}^{q}\left(\text { see }(4.23),(4.24) \text { and }\left(\mathbf{H}_{2}\right)(i v)\right) \\
& =t^{q}\left[\varepsilon\|D \widetilde{u}\|_{q}^{q}-\widehat{C}_{0}\|\widetilde{u}\|_{q}^{q}\right] .
\end{aligned}
$$

If we choose $\varepsilon \in\left(0, \frac{\widehat{C}_{0}\|\widetilde{u}\|_{q}^{q}}{\|D \widetilde{u}\|_{q}^{q}}\right)$ then from (4.25) we infer that

$$
\varphi_{+}^{\lambda}(t \widetilde{u})<0
$$

and so, (4.22) is true.

Now, let

$$
\gamma=\inf _{\partial B_{\rho_{\lambda}^{+}}} \varphi_{+}^{\lambda}-\inf _{B_{\rho_{\lambda}^{+}}} \varphi_{+}^{\lambda}>0
$$

(see (4.22) and Proposition 6). Here

$$
\bar{B}_{\rho_{\lambda}^{+}}=\left\{u \in W_{0}^{1, p}(\Omega):\|u\| \leq \rho_{\lambda}^{+}\right\} \text {and } \partial B_{\rho_{\lambda}^{+}}=\left\{u \in W_{0}^{1, p}(\Omega):\|u\|=\rho_{\lambda}^{+}\right\} .
$$


Invoking the Ekeland variational principle with $\varepsilon \in(0, \gamma)$ (see, for example, GasinskiPapageorgiou [20], p. 579), we can find $u_{\varepsilon} \in \bar{B}_{\rho_{\lambda}^{+}}$such that

$$
\varphi_{+}^{\lambda}\left(u_{\varepsilon}\right) \leq \inf _{\bar{B}_{\rho_{\lambda}^{+}}} \varphi_{+}^{\lambda}+\varepsilon
$$

and

$$
\varphi_{+}^{\lambda}\left(u_{\varepsilon}\right) \leq \varphi_{+}^{\lambda}(u)+\varepsilon\left\|u-u_{\varepsilon}\right\| \text { for all } u \in \bar{B}_{\rho_{\lambda}^{+}} .
$$

Since $\varepsilon \in(0, \gamma)$, from $(4.26)$ it follows that

$$
\varphi_{+}^{\lambda}\left(u_{\varepsilon}\right)<\inf _{\partial B_{\rho_{\lambda}^{+}}} \varphi_{+}^{\lambda},
$$

hence

$$
u_{\varepsilon} \in B_{\rho_{\lambda}^{+}}=\left\{u \in W_{0}^{1, p}(\Omega):\|u\|<\rho_{\lambda}^{+}\right\}
$$

Let $h \in W_{0}^{1, p}(\Omega)$ and let $u=u_{\varepsilon}+t h$ with $t>0$. Because of (4.28), for $t>0$ small we have $u \in \bar{B}_{\rho_{\lambda}^{+}}$and so, from (4.27) it follows that

$$
-\varepsilon\|h\| \leq\left\langle\left(\varphi_{+}^{\lambda}\right)^{\prime}\left(u_{\varepsilon}\right), h\right\rangle \text { for all } h \in W_{0}^{1, p}(\Omega),
$$

hence

$$
\left\|\left(\varphi_{+}^{\lambda}\right)^{\prime}\left(u_{\varepsilon}\right)\right\|_{*} \leq \varepsilon
$$

Let $\varepsilon_{n}=\frac{1}{n}$ and $u_{n}=u_{\varepsilon_{n}}$, for $n \geq 1$. Then

$$
\varphi_{+}^{\lambda}\left(u_{n}\right) \rightarrow \inf _{\bar{B}_{\rho_{\lambda}^{+}}} \varphi_{+}^{\lambda}(\operatorname{see}(4.26))
$$

and

$$
\left(\varphi_{+}^{\lambda}\right)^{\prime}\left(u_{n}\right) \rightarrow 0 \text { in } W^{-1, p^{\prime}}(\Omega)(\text { see }(4.29)) .
$$

Since $\left\{u_{n}\right\}_{n \geq 1} \subseteq \bar{B}_{\rho_{\lambda}^{+}}$(see (4.28)) and because $\varphi_{+}^{\lambda}$ satisfies the C-condition (see Proposition 4), from (4.30) and (4.31) it follows that at least for a subsequence, we have

$$
u_{n} \rightarrow \widehat{u} \text { in } W_{0}^{1, p}(\Omega),
$$

hence

$$
\varphi_{+}^{\lambda}(\widehat{u})=\inf _{\bar{B}_{\rho_{\lambda}^{+}}} \varphi_{+}^{\lambda} \text { and }\left(\varphi_{+}^{\lambda}\right)^{\prime}(\widehat{u})=0 .
$$

From (4.22), we see that

$$
\varphi_{+}^{\lambda}(\widehat{u})<0=\varphi_{+}^{\lambda}(0),
$$


i.e.,

$$
\widehat{u} \neq 0,\|\widehat{u}\| \leq \rho_{\lambda}^{+}
$$

Also from (4.32), we have

$$
A(\widehat{u})=N_{f_{+}^{\lambda}}(\widehat{u}), \text { where } f_{+}^{\lambda}(z, x)=f_{+}(z, x, \lambda) .
$$

On (4.33) we act with $-\widehat{u}^{-} \in W_{0}^{1, p}(\Omega)$ and obtain $\widehat{u} \geq 0, \widehat{u} \neq 0$. We have

$$
-\operatorname{div} a(z, D \widehat{u}(z))=f(z, \widehat{u}(z), \lambda) \text { a.e. in } \Omega,\left.\widehat{u}\right|_{\partial \Omega}=0 \text {. }
$$

From nonlinear regularity theory (see [26], [28]) we infer that $\widehat{u} \in C_{+} \backslash\{0\}$. From (4.34) and hypothesis $\left(\mathbf{H}_{2}\right)(i v)$, we have

$$
-\operatorname{div} a(z, D \widehat{u}(z)) \geq 0 \text { a.e. in } \Omega,
$$

and since $f(., \widehat{u}(),. \lambda) \neq 0$, from Theorem 2.1 of Cuesta-Takac [14] it follows that $\widehat{u} \in \operatorname{int} C_{+}$.

Note that

$$
\left.\varphi_{\lambda}\right|_{C_{+}}=\left.\varphi_{+}^{\lambda}\right|_{C_{+}}
$$

Hence $\widehat{u}$ is a local $C_{0}^{1}(\Omega)-$ minimizer of $\varphi_{\lambda}$. Invoking Proposition 2 , it follows that $\widehat{u}$ is a local $W_{0}^{1, p}(\Omega)-$ minimizer of $\varphi_{\lambda}$.

Hypothesis $\left(\mathbf{H}_{2}\right)$ (ii) implies that for $\widetilde{u} \in \operatorname{int} C_{+}$, we have

$$
\varphi_{+}^{\lambda}(t \widetilde{u}) \rightarrow-\infty \text { as } t \rightarrow \infty .
$$

Then (4.35) and Propositions 4 and 6 permit the use of Theorem 1 (the mountain pass theorem). So, we can find $u_{0} \in W_{0}^{1, p}(\Omega)$ such that

$$
\varphi_{+}^{\lambda}(\widehat{u})<0=\varphi_{+}^{\lambda}(0)<\eta_{+}^{\lambda} \leq \varphi_{+}^{\lambda}\left(u_{0}\right)
$$

and

$$
\left(\varphi_{+}^{\lambda}\right)^{\prime}\left(u_{0}\right)=0
$$

From (4.36) we see that $u_{0} \neq \widehat{u}$ and $u_{0} \neq 0$. From (4.37) we have

$$
A\left(u_{0}\right)=N_{f_{+}^{\lambda}}\left(u_{0}\right) .
$$

Acting on (4.38) with $-u_{0}^{-} \in W_{0}^{1, p}(\Omega)$, we obtain $u_{0} \geq 0, u_{0} \neq 0$. We have

$$
-\operatorname{div} a\left(z, D u_{0}(z)\right)=f\left(z, u_{0}(z), \lambda\right) \text { a.e. in } \Omega,\left.u_{0}\right|_{\partial \Omega}=0 \text {. }
$$

As before, nonlinear regularity (see [26], [28]) and Theorem 2.1 of [14] together with $\left(\mathbf{H}_{2}\right)(i v)$ imply that $u_{0} \in$ int $C_{+}$. $\varphi_{-}^{\lambda}$.

(b) The proof of this part is similar to that of $(a)$, using this time the functional

(c) This part is a direct consequence of $(a)$ and $(b)$. 
5. Nodal solutions. In this section we look for nodal (sign-changing) solutions. The idea is to look for extremal constant sign solutions, i.e., a smallest nontrivial positive solution $u_{*}$ and a biggest nontrivial negative solution $v_{*}$, then look for a nontrivial solution distinct from $u_{*}, v_{*}$ in the order interval

$$
\left[v_{*}, u_{*}\right]:=\left\{u \in W_{0}^{1, p}(\Omega): v_{*}(z) \leq u(z) \leq u_{*}(z) \text { a.e. in } \Omega\right\} .
$$

Evidently, such a solution is necessarily nodal. The lack of homogeneity in the differential operator, creates difficulties in the implementation of this strategy and in particular in establishing the existence of extremal constant sign solutions. To overcome these difficulties we consider the following auxiliary Dirichlet problem

$$
-\operatorname{div} a(z, D u(z))=\widehat{C}_{0}|u(z)|^{q-2} u(z) \text { a.e. in } \Omega,\left.u\right|_{\partial \Omega}=0 \text {. }
$$

Proposition 8. If hypotheses $\left(\mathbf{H}_{0}\right)$ hold, then problem (5.1) has a unique nontrivial positive solution $\bar{u} \in$ int $C_{+}$, and, by oddness of (5.1), $\bar{v}=-\bar{u} \in-$ int $C_{+}$is the unique nontrivial negative solution of (5.1).

Proof. Let $\psi_{+}: W_{0}^{1, p}(\Omega) \rightarrow \mathbb{R}$ be the $C^{1}$-functional defined by

$$
\psi_{+}(u)=\int_{\Omega} G(z, D u(z)) d z-\frac{\widehat{C}_{0}}{q}\left\|u^{+}\right\|_{q}^{q} \text { for all } u \in W_{0}^{1, p}(\Omega) .
$$

From (3.4), we have

$$
\psi_{+}(u) \geq \frac{C_{1}}{p(p-1)}\|u\|^{p}-C_{13}\|u\|^{q} \text { for some } C_{13}>0, \text { all } u \in W_{0}^{1, p}(\Omega) .
$$

Because $q<p$, from (5.3) it follows that $\psi_{+}$is coercive. Moreover, using the Sobolev embedding theorem, we see that $\psi_{+}$is sequentially weakly lower semicontinuous. So, by the Weierstrass theorem, we can find $\bar{u} \in W_{0}^{1, p}(\Omega)$ such that

$$
\psi_{+}(\bar{u})=\inf \left\{\psi_{+}(u): u \in W_{0}^{1, p}(\Omega)\right\}=: \bar{m}_{+} .
$$

As in the proof of Proposition 7 (see (4.25)), we show that

$$
\psi_{+}(\bar{u})=\bar{m}_{+}<0=\psi_{+}(0), \text { i.e., } \bar{u} \neq 0 .
$$

From (5.4) we have

$$
\left(\psi_{+}\right)^{\prime}(\bar{u})=0
$$

hence

$$
A(\bar{u})=\widehat{C}_{0}\left(\bar{u}^{+}\right)^{q-1} .
$$

Acting in (5.5) with $-\bar{u}^{-} \in W_{0}^{1, p}(\Omega)$, we obtain $\bar{u} \geq 0, \bar{u} \neq 0$. Moreover, as before, nonlinear regularity (see [26], [28]) and Theorem 2.1 of [14], imply that $\bar{u} \in$ int $C_{+}$.

Evidently, due to the oddness of $(5.1), \bar{v}=-\bar{u} \in-i n t C_{+}$is a nontrivial negative solution of (5.1).

Finally, the uniqueness of these constant sign solutions follows from Proposition 3 noting that $x \rightarrow \frac{\widehat{C}_{0} x^{q-1}}{x^{\tau-1}}$ is strictly decreasing on $(0, \infty)$ and $x \rightarrow \frac{\widehat{C}_{0}|x|^{q-2} x}{|x|^{\tau-2} x}$ is strictly increasing on $(-\infty, 0)$ (recall that $\tau \in(q, p)$ ). 
This proposition leads to the existence of extremal constant sign solutions for problem $\left(P_{\lambda}\right)$.

Proposition 9. If hypotheses $\left(\mathbf{H}_{0}\right)$ and $\left(\mathbf{H}_{2}\right)$ hold and $\lambda \in\left(0, \lambda^{*}\right)$, then problem $\left(P_{\lambda}\right)$ has a smallest nontrivial positive solution $u_{*} \in$ int $C_{+}$and a biggest nontrivial negative solution $v_{*} \in-$ int $C_{+}$.

Proof. For $\lambda \in\left(0, \lambda^{*}\right)$, let $\mathcal{S}_{+}^{\lambda}$ be the set of nontrivial positive solutions of problem $\left(P_{\lambda}\right)$. From Proposition 7 and its proof, we know that

$$
\mathcal{S}_{+}^{\lambda} \neq \varnothing \text { and } \mathcal{S}_{+}^{\lambda} \subseteq \operatorname{int} C_{+} .
$$

Claim. If $\widetilde{u} \in \mathcal{S}_{+}^{\lambda}$, then $\widetilde{u} \geq \bar{u}$.

We consider the following Carathéodory function

$$
\mu_{+}(z, x)= \begin{cases}0 & \text { if } \quad x<0 \\ \widehat{C}_{0} x^{q-1} & \text { if } \quad 0 \leq x \leq \widetilde{u}(z) \\ \widehat{C}_{0} \widetilde{u}(z)^{q-1} & \text { if } \quad \widetilde{u}(z)<x\end{cases}
$$

We set $M_{+}(z, x)=\int_{0}^{x} \mu_{+}(z, s) d s$ and then introduce the $C^{1}$-functional $\xi_{+}$: $W_{0}^{1, p}(\Omega) \rightarrow \mathbb{R}$ defined by

$$
\xi_{+}(u)=\int_{\Omega} G(z, D u(z)) d z-\int_{\Omega} M_{+}(z, u(z)) d z .
$$

It is clear from (5.6) that $\xi_{+}$is coercive. Also, it is sequentially weakly lower semicontinuous. Thus, we can find $y_{0} \in W_{0}^{1, p}(\Omega)$ such that

$$
\xi_{+}\left(y_{0}\right)=\inf \left\{\xi_{+}(y): y \in W_{0}^{1, p}(\Omega)\right\}=: m_{+}^{0} .
$$

As before (see the proof of Proposition 7), since $q<p$, we see that

$$
m_{+}^{0}=\xi_{+}\left(y_{0}\right)<0=\xi_{+}(0) \text {, i.e., } y_{0} \neq 0 .
$$

From (5.7), we have

$$
\left(\xi_{+}\right)^{\prime}\left(y_{0}\right)=0
$$

hence

$$
A\left(y_{0}\right)=N_{\mu_{+}}\left(y_{0}\right) .
$$

On (5.8) we act with $-y_{0}^{-} \in W_{0}^{1, p}(\Omega)$, we obtain $y_{0} \geq 0, y_{0} \neq 0$. Also, on (5.8) we act with $\left(y_{0}-\widetilde{u}\right)^{+} \in W_{0}^{1, p}(\Omega)$. Then

$$
\begin{aligned}
\left\langle A\left(y_{0}\right),\left(y_{0}-\widetilde{u}\right)^{+}\right\rangle & =\int_{\Omega} \mu_{+}\left(z, y_{0}\right)\left(y_{0}-\widetilde{u}\right)^{+} d z \\
& =\widehat{C}_{0} \int_{\Omega} \widetilde{u}^{q-1}\left(y_{0}-\widetilde{u}\right)^{+} d z(\text { see }(5.6)) \\
& \leq \int_{\Omega} f(z, \widetilde{u}, \lambda)\left(y_{0}-\widetilde{u}\right)^{+} d z\left(\operatorname{see}\left(\mathbf{H}_{2}\right)(i v)\right) \\
& =\left\langle A(\widetilde{u}),\left(y_{0}-\widetilde{u}\right)^{+}\right\rangle,
\end{aligned}
$$


hence

$$
\int_{\left\{y_{0}>\widetilde{u}\right\}}\left(a\left(z, D y_{0}\right)-a(z, D \widetilde{u}), D y_{0}-D \widetilde{u}\right)_{\mathbb{R}^{N}} d z \leq 0
$$

therefore

$$
\left|\left\{y_{0}>\widetilde{u}\right\}\right|_{N}=0 \text {, i.e., } y_{0} \leq \widetilde{u} .
$$

Hence, we have proved that $y_{0} \in[0, \widetilde{u}] \backslash\{0\}$. Then (5.8) becomes

$$
A\left(y_{0}\right)=\widehat{C}_{0} y_{0}^{p-1}(\text { see }(5.6)),
$$

hence

$$
-\operatorname{div} a\left(z, D y_{0}(z)\right)=\widehat{C}_{0} y_{0}(z)^{p-1} \text { a.e. in } \Omega,\left.y_{0}\right|_{\partial \Omega}=0
$$

therefore

$$
y_{0}=\bar{u} \in \operatorname{int} C_{+}(\text {see Proposition 8) }
$$

and we conclude

$$
\bar{u} \leq \widetilde{u}
$$

This proves the Claim.

Next, let $C \subseteq \mathcal{S}_{+}^{\lambda}$ be a chain (i.e., a totally ordered subset of $\mathcal{S}_{+}^{\lambda}$ ). Invoking Dunford-Schwartz ([16], p.136), we can find $\left\{u_{n}\right\}_{n \geq 1} \subset C$ such that

$$
\text { inf } C=\inf _{n \geq 1} u_{n} .
$$

Moreover, Lemma 1.1.5 of Heikkila-Lakshmikantam [22] implies that we can choose $\left\{u_{n}\right\}_{n \geq 1} \subset C$ to be decreasing. We have

$$
A\left(u_{n}\right)=N_{f_{\lambda}}\left(u_{n}\right) \text { for all } n \geq 1 \text {, where } f_{\lambda}(z, x)=f(z, x, \lambda),
$$

hence

$$
\left\{u_{n}\right\}_{n \geq 1} \subseteq W_{0}^{1, p}(\Omega) \text { is bounded. }
$$

So, we may assume that

$$
u_{n} \stackrel{w}{\longrightarrow} u \text { in } W_{0}^{1, p}(\Omega) \text { and } u_{n} \rightarrow u \text { in } L^{r}(\Omega) \text { as } n \rightarrow \infty .
$$

On (5.9) we act with $u_{n}-u$, pass to the limit as $n \rightarrow \infty$ and use (5.10). It follows that

$$
\lim _{n \rightarrow \infty}\left\langle A\left(u_{n}\right), u_{n}-u\right\rangle=0
$$

hence

$$
\left.u_{n} \rightarrow u \text { in } W_{0}^{1, p}(\Omega) \text { as } n \rightarrow \infty \text { (see Proposition } 1\right) .
$$


So, if in (5.9) we pass to the limit as $n \rightarrow \infty$ and use (5.11), we conclude that

$$
A(u)=N_{f_{\lambda}}(u),
$$

hence (see the Claim)

$$
u \in \mathcal{S}_{+}^{\lambda} \text { and } u=\inf C .
$$

Since $C \subseteq \mathcal{S}_{+}^{\lambda}$ is an arbitrary chain, invoking the Kuratowski-Zorn lemma, we infer that $\mathcal{S}_{+}^{\lambda}$ has a minimal element $u_{*} \in$ int $C_{+}$. As in Aizicovici-Papageorgiou-Staicu[2] (see Lemma 1 and the proof of Proposition 8), we show that $\mathcal{S}_{+}^{\lambda}$ is downward directed (i.e., if $u_{1}, u_{2} \in \mathcal{S}_{+}^{\lambda}$, then we can find $u \in \mathcal{S}_{+}^{\lambda}$ such that $u \leq u_{1}, u \leq u_{2}$ ). Therefore $u_{*} \in$ int $C_{+}$is the smallest nontrivial positive solution of $\left(P_{\lambda}\right)$.

Similarly, let $\mathcal{S}_{-}^{\lambda}$ be the set of nontrivial negative solutions of $\left(P_{\lambda}\right)\left(\lambda \in\left(0, \lambda^{*}\right)\right)$. From Proposition 7 and its proof, we know that

$$
\mathcal{S}_{-}^{\lambda} \neq \varnothing \text { and } \mathcal{S}_{-}^{\lambda} \subseteq-i n t C_{+} .
$$

Moreover, $\mathcal{S}_{-}^{\lambda}$ is upward directed (i.e., if $v_{1}, v_{2} \in \mathcal{S}_{-}^{\lambda}$, then we can find $v \in \mathcal{S}_{-}^{\lambda}$ such that $v_{1} \leq v, v_{2} \leq v$; see [2], Lemma 2). So, as for $\mathcal{S}_{+}^{\lambda}$, we can establish the existence of the biggest nontrivial negative solution $v_{*} \in-i n t C_{+}$of $\left(P_{\lambda}\right)$.

Now we are ready to produce a nodal solution.

Proposition 10. If hypotheses $\left(\mathbf{H}_{0}\right)$ and $\left(\mathbf{H}_{2}\right)$ hold and $\lambda \in\left(0, \lambda^{*}\right)$, then problem $\left(P_{\lambda}\right)$ admits a nodal solution $y_{0} \in C_{0}^{1}(\bar{\Omega})$.

Proof. Let $u_{*} \in$ int $C_{+}$and $v_{*} \in-$ int $C_{+}$be the two extremal constant sign solutions of problem $\left(P_{\lambda}\right)\left(\lambda \in\left(0, \lambda^{*}\right)\right)$ produced in Proposition 9. We introduce the following truncation of the reaction $f(z, ., \lambda)$ :

$$
h(z, x, \lambda)=\left\{\begin{array}{lll}
f\left(z, v_{*}(z), \lambda\right) & \text { if } & x<v_{*}(z) \\
f(z, x, \lambda) & \text { if } & v_{*}(z) \leq x \leq u_{*}(z) \\
f\left(z, u_{*}(z), \lambda\right) & \text { if } & u_{*}(z)<x
\end{array}\right.
$$

This is a Carathéodory function. Let $H(z, x, \lambda)=\int_{0}^{x} h(z, s, \lambda) d s$ and consider the $C^{1}$-functional $\widehat{\psi}_{\lambda}: W_{0}^{1, p}(\Omega) \rightarrow \mathbb{R}$ defined by

$$
\widehat{\psi}_{\lambda}(u)=\int_{\Omega} G(z, D u(z)) d z-\int_{\Omega} H(z, u(z), \lambda) d z \text { for all } u \in W_{0}^{1, p}(\Omega) .
$$

Also, let $h_{ \pm}(z, x, \lambda)=h\left(z, \pm x^{ \pm}, \lambda\right), H_{ \pm}(z, x, \lambda)=\int_{0}^{x} h_{ \pm}(z, s, \lambda) d s$ and consider the $C^{1}$-functional $\widehat{\psi}_{ \pm}^{\lambda}: W_{0}^{1, p}(\Omega) \rightarrow \mathbb{R}$ defined by

$$
\widehat{\psi}_{ \pm}^{\lambda}(u)=\int_{\Omega} G(z, D u(z)) d z-\int_{\Omega} H_{ \pm}(z, u(z), \lambda) d z \text { for all } u \in W_{0}^{1, p}(\Omega) .
$$

Using (5.12) and reasoning as in the proof of Proposition 9, we obtain

$$
K_{\widehat{\psi}_{\lambda}} \subseteq\left[v_{*}, u_{*}\right], K_{\widehat{\psi}_{+}^{\lambda}} \subseteq\left[0, u_{*}\right], K_{\widehat{\psi}_{-}^{\lambda}} \subseteq\left[v_{*}, 0\right] .
$$


In fact the extremality of $v_{*}, u_{*}$ and (5.12) imply that

$$
K_{\widehat{\psi}_{\lambda}} \subseteq\left[v_{*}, u_{*}\right], K_{\widehat{\psi}_{+}^{\lambda}}=\left\{0, u_{*}\right\}, K_{\widehat{\psi}_{-}^{\lambda}}=\left\{v_{*}, 0\right\}
$$

Claim. $u_{*} \in \operatorname{int} C_{+}$and $v_{*} \in-$ int $C_{+}$are local minimizers of $\widehat{\psi}_{\lambda}$.

From (5.12) it is clear that $\widehat{\psi}_{+}^{\lambda}$ is coercive. Also, it is sequentially weakly lower semicontinuous. Therefore, we can find $w_{+} \in W_{0}^{1, p}(\Omega)$ such that

$$
\widehat{\psi}_{+}^{\lambda}\left(w_{+}\right)=\inf \left\{\widehat{\psi}_{+}^{\lambda}(w): w \in W_{0}^{1, p}(\Omega)\right\} .
$$

As before (see the proof of Proposition 7), using $\left(\mathbf{H}_{2}\right)(i v)$ and the fact that $q<p$ we have

$$
\widehat{\psi}_{+}^{\lambda}\left(w_{+}\right)<0=\widehat{\psi}_{+}^{\lambda}(0)
$$

hence

$$
w_{+} \neq 0, \text { and so, } w_{+}=u_{*}(\operatorname{see}(5.13)) .
$$

Recall that $u_{*} \in$ int $C_{+}$(see Proposition 9) and note that

$$
\left.\widehat{\psi}_{\lambda}\right|_{C_{+}}=\left.\widehat{\psi}_{+}^{\lambda}\right|_{C_{+}} .
$$

Therefore $u_{*}$ is a local $C_{0}^{1}(\bar{\Omega})$ minimizer of $\widehat{\psi}_{\lambda}$. Invoking Proposition 2 , we conclude that $u_{*} \in$ int $C_{+}$is a local $W_{0}^{1, p}(\Omega)$ minimizer of $\widehat{\psi}_{\lambda}$. Similary, if we use $\widehat{\psi}_{-}^{\lambda}$, then we show that $v_{*} \in-i n t C_{+}$is a local $W_{0}^{1, p}(\Omega)$ minimizer of $\widehat{\psi}_{\lambda}$.

Without any loss of generality, we may assume that

$$
\widehat{\psi}_{\lambda}\left(v_{*}\right) \leq \widehat{\psi}_{\lambda}\left(u_{*}\right)
$$

(the analysis is similar, if the opposite inequality holds). Using the Claim and reasoning as in Aizicovici-Papageorgiou-Staicu [1] (see the proof of Proposition 29), we can find $\rho_{\lambda} \in(0,1)$ small such that

$$
\widehat{\psi}_{\lambda}\left(v_{*}\right) \leq \widehat{\psi}_{\lambda}\left(u_{*}\right)<\inf \left\{\widehat{\psi}_{\lambda}(u):\left\|u-u_{*}\right\|=\rho_{\lambda}\right\}=\widehat{\eta}_{\lambda}
$$

Since $\widehat{\psi}_{\lambda}$ is coercive (see $(5.12)$ ), it satisfies the C-condition. This fact and (5.15) permit the use of Theorem 1 (the mountain pass theorem). So, we can find $y_{0} \in$ $K_{\widehat{\psi}_{\lambda}} \subseteq\left[v_{*}, u_{*}\right]($ see $(5.13))$ such that

$$
\widehat{\psi}_{\lambda}\left(v_{*}\right) \leq \widehat{\psi}_{\lambda}\left(u_{*}\right)<\widehat{\eta}_{\lambda} \leq \widehat{\psi}_{\lambda}\left(y_{0}\right),
$$

hence

$$
y_{0} \notin\left\{v_{*}, u_{*}\right\} .
$$

Since $y_{0} \in K_{\widehat{\psi}_{\lambda}} \subseteq\left[v_{*}, u_{*}\right]$ and

$$
\left.\widehat{\psi}_{\lambda}^{\prime}\right|_{\left[v_{*}, u_{*}\right]}=\left.\varphi_{\lambda}^{\prime}\right|_{\left[v_{*}, u_{*}\right]}
$$


(see (5.12)), we see that $y_{0}$ solves $\left(P_{\lambda}\right)$ and $y_{0} \in C_{0}^{1}(\bar{\Omega})$ (nonlinear regularity). Since $y_{0}$ is a critical point of $\widehat{\psi}_{\lambda}$ of mountain pass type, we have

$$
C_{1}\left(\widehat{\psi}_{\lambda}, y_{0}\right) \neq 0 \text { (see Chang [11], p. 89). }
$$

On the other hand, hypothesis $\left(\mathbf{H}_{2}\right)(i v)$ and Proposition 2.1 of Jiu-Su [24] imply that

$$
C_{k}\left(\widehat{\psi}_{\lambda}, 0\right)=0 \text { for all } k \geq 0
$$

Comparing (5.16) and (5.17) we infer that $y_{0} \neq 0$. Since $y_{0} \in\left[v_{*}, u_{*}\right], y_{0} \notin\left\{0, v_{*}, u_{*}\right\}$, by virtue of extremality of the solution $v_{*}, u_{*}$, we conclude that $y_{0}$ is nodal.

So, summarizing the situation for problem $\left(P_{\lambda}\right)$, we can state the following multiplicity theorem.

THEOREM 2. If hypotheses $\left(\mathbf{H}_{0}\right)$ and $\left(\mathbf{H}_{2}\right)$ hold, then there exists $\lambda^{*}>0$ such that for all $\lambda \in\left(0, \lambda^{*}\right)$, problem $\left(P_{\lambda}\right)$ has at least five nontrivial smooth solutions $u_{0}, \widehat{u} \in$ int $C_{+}, v_{0}, \widehat{v} \in-$ int $C_{+}$and $y_{0} \in C_{0}^{1}(\bar{\Omega}) \backslash\{0\}$, nodal.

6. Hilbert space case $(p=2)$. In this section, we consider the Hilbert space case (i.e., $p=2$, hence the ambient space is $H_{0}^{1}(\Omega)$ ) and under stronger differentiability conditions on $a(z,$.$) , we show that for all \lambda \in\left(0, \lambda^{*}\right)$, problem $\left(P_{\lambda}\right)$ has at least six nontrivial smooth solutions: two positive, two negative, one nodal and a sixth one for which we cannot determine its sign.

In this case $h \in C^{1}(0, \infty)$ satisfies

$$
0<\frac{t h^{\prime}(t)}{h(t)} \leq C_{0} \text { for all } t>0 \text { and some } C_{0}>0
$$

and

$$
C_{1} t \leq h(t) \leq C_{2}\left(t^{q_{0}-1}+t\right) \text { for all } t>0 \text { and some } C_{1}, C_{2}>0,1<q_{0} \leq 2
$$

(see (3.1) with $p=2$ ). The new stronger hypotheses on $a(z, y)$ are the following:

$\left(\mathbf{H}_{0}^{\prime}\right) a(z, y)=a_{0}(z,\|y\|) y$ where $a_{0}(z, t)>0$ for all $(z, t) \in \bar{\Omega} \times(0, \infty)$ and

(i) $a \in C^{1}\left(\bar{\Omega} \times \mathbb{R}^{N}, \mathbb{R}^{N}\right), \lim _{t \rightarrow 0^{+}} a_{0}(z, t) t=0$ for all $z \in \bar{\Omega}$;

(ii) for all $(z, y) \in \bar{\Omega} \times \mathbb{R}^{N}$ :

$$
\frac{h(\|y\|)}{\|y\|}\|\xi\|^{2} \leq\left(\nabla_{y} a(z, y) \xi, \xi\right)_{\mathbb{R}^{N}} \text { for all } \xi \in \mathbb{R}^{N}
$$

(iii) for all $(z, y) \in \bar{\Omega} \times \mathbb{R}^{N}$ we have

$$
\left\|\nabla_{y} a(z, y)\right\| \leq C_{3} \frac{h(\|y\|)}{\|y\|} \text { for some } C_{3}>0 ;
$$

(iv) the primitive $G(z, y)$ determined by

$$
\begin{aligned}
& \nabla_{y} G(z, y)=a(z, y) \text { for all }(z, y) \in \bar{\Omega} \times \mathbb{R}^{N} \text { and } \\
& G(z, 0)=0 \text { for all } z \in \bar{\Omega}
\end{aligned}
$$

satisfies

$$
\begin{aligned}
& k(z) \leq p G(z, y)-(a(z, y), y)_{\mathbb{R}^{N}} \text { for a.a. } z \in \Omega, \text { all } y \in \mathbb{R}^{N}, \\
& \quad \text { with } k \in L^{1}(\Omega)
\end{aligned}
$$


$(v)$ there exists $q \in(1,2)$ such that

$$
\lim _{y \rightarrow 0} \frac{G(z, y)}{\|y\|^{q}}=0 \text { uniformly for all } z \in \bar{\Omega}
$$

and if

$$
G_{0}(z, t)=\int_{0}^{t} a_{0}(z, s) s d s, \text { fort }>0
$$

then for some $\tau \in(q, 2), t \rightarrow G_{0}\left(z, t^{\frac{1}{\tau}}\right)$ is convex.

We also strengthen the hypotheses on the reaction $f(x, z, \lambda)$ :

$\left(\mathbf{H}_{2}^{\prime}\right): f: \Omega \times \mathbb{R} \times(0, \infty) \rightarrow \mathbb{R}$ is a function such that for all $\lambda>0,(z, x) \rightarrow f(z, x, \lambda)$ is a measurable, for a.a. $z \in \Omega, f(z, ., \lambda) \in C^{1}(\mathbb{R})$ and is nondecreasing, $f(z, 0, \lambda)=0$ for a.a. $z \in \Omega$ and

(i) $\left|f_{x}^{\prime}(z, x, \lambda)\right| \leq \alpha(z, \lambda)+C(\lambda)|x|^{r-2}$ for a.a. $z \in \Omega$, all $x \in \mathbb{R}$ with $\alpha(., \lambda) \in L^{\infty}(\Omega)_{+}, C(\lambda)>0$ and $2<r<2^{*}$;

(ii) for $F(z, x, \lambda)=\int_{0}^{x} f(z, s, \lambda) d s$ we have

$$
\lim _{x \rightarrow \pm \infty} \frac{F(z, x, \lambda)}{|x|^{2}}=+\infty \text { uniformly for a.a. } z \in \Omega
$$

(iii) for every $\lambda>0$, there exists $\tau_{0}=\tau_{0}(\lambda) \in\left((r-2) \max \left\{1, \frac{N}{2}\right\}, 2^{*}\right)$ and $\beta_{0}=\beta_{0}(\lambda)>0$ such that

$$
\beta_{0} \leq \liminf _{x \rightarrow \pm \infty} \frac{f(z, x, \lambda) x-2 F(z, x, \lambda)}{|x|^{\tau_{0}}} \text { uniformly for a.a. } z \in \Omega
$$

(iv) if $q \in(1,2)$ is as in hypothesis $\left(\mathbf{H}_{0}^{\prime}\right)(v)$, then for all $\lambda>0$ we have

$$
\begin{aligned}
\widehat{C}_{0}|x|^{q} & \leq f(z, x, \lambda) x \text { for a.a. } z \in \Omega, \text { all } x \in \mathbb{R}, \\
\text { with } \widehat{C}_{0} & =\widehat{C}_{0}(\lambda)>0,
\end{aligned}
$$

there exists $\delta_{0}=\delta_{0}(\lambda)>0$ such that

$$
\begin{aligned}
& 0<f(z, x, \lambda) x \leq q F(z, x, \lambda) \text { for a.a. } z \in \Omega \text {, all }|x| \leq \delta_{0} \text {, } \\
& \text { ess inf } F\left(., \delta_{0}, \lambda\right)>0 \text {, } \\
& \Omega
\end{aligned}
$$

and there exists $\eta_{0}=\eta_{0}(., \lambda) \in L^{\infty}(\Omega)_{+}$with $\left\|\eta_{0}(., \lambda)\right\|_{\infty} \rightarrow 0$ as $\lambda \rightarrow 0^{+}$and

$$
\limsup _{x \rightarrow \pm \infty} \frac{F(z, x, \lambda)}{|x|^{q}} \leq \eta_{0}(z, \lambda) \text { uniformly for a.a. } z \in \Omega .
$$

In this case, for every $\lambda>0, \varphi_{\lambda} \in C^{2}\left(H_{0}^{1}(\Omega)\right)$.

Theorem 3. If hypotheses $\left(\mathbf{H}_{0}^{\prime}\right)$ and $\left(\mathbf{H}_{2}^{\prime}\right)$ hold, then there exists $\lambda^{*}>0$ such that for all $\lambda \in\left(0, \lambda^{*}\right)$, problem $\left(P_{\lambda}\right)$ has at least six nontrivial smooth solutions $u_{0}, \widehat{u} \in$ int $C_{+}, v_{0}, \widehat{v} \in-$ int $C_{+}, y_{0} \in C_{0}^{1}(\bar{\Omega}) \backslash\{0\}$ nodal, and $\widehat{y} \in C_{0}^{1}(\bar{\Omega}) \backslash\{0\}$. 
Proof. From Theorem 2 we know that we can find $\lambda^{*}>0$ such that for all $\lambda \in\left(0, \lambda^{*}\right)$, problem $\left(P_{\lambda}\right)$ has at least five nontrivial smooth solutions $u_{0}, \widehat{u} \in \operatorname{int} C_{+}$, $v_{0}, \widehat{v} \in-$ int $C_{+}$, and $y_{0} \in C_{0}^{1}(\bar{\Omega}) \backslash\{0\}$ nodal.

Let $u_{*} \in$ int $C_{+}$and $v_{*} \in$ int $C_{+}$be the extremal constant sign solutions produced in Proposition 9. Since $y_{0}$ is nodal, $y_{0}^{+} \neq 0$ and so $f\left(., y_{0}^{+}(),. \lambda\right) \neq 0$ (see $\left.\left(\mathbf{H}_{2}^{\prime}\right)(i v)\right)$. Let $\widehat{w}_{0} \in$ int $C_{+}$be the unique solution of

$$
-\operatorname{div} a(z, D \widehat{w}(z))=f\left(z, y_{0}^{+}(z), \lambda\right) \text { in } \Omega,\left.\widehat{w}\right|_{\partial \Omega}=0 .
$$

Recalling that $y_{0} \leq u_{*}$, hence $y_{0}^{+} \leq u_{*}$, we have

$$
A\left(\widehat{w}_{0}\right)=N_{f_{\lambda}}\left(y_{0}^{+}\right) \leq N_{f_{\lambda}}\left(u_{*}\right)=A\left(u_{*}\right) .
$$

Because $f\left(., y_{0}^{+}(),. \lambda\right) \neq f\left(., u_{*}(),. \lambda\right)$ (recall that $y_{0}$ is nodal and $u_{*} \in \operatorname{int} C_{+}$), from (6.1) and Theorem 2.1 of Cuesta-Takac [14] it follows that $u_{*}-y_{0} \in$ int $C_{+}$. Similarly we show that $y_{0}-v_{*} \in$ int $C_{+}$. Therefore

$$
y_{0} \in \operatorname{int}_{C_{0}^{1}(\bar{\Omega})}\left[v_{*}, u_{*}\right] .
$$

Let $\widehat{\psi}_{\lambda}$ be as in the proof of Proposition 10. We have

$$
\left.\widehat{\psi}_{\lambda}\right|_{\left[v_{*}, u_{*}\right]}=\left.\varphi_{\lambda}\right|_{\left[v_{*}, u_{*}\right]}(\operatorname{see}(5.12))
$$

hence

$$
C_{k}\left(\left.\widehat{\psi}_{\lambda}\right|_{C_{0}^{1}(\bar{\Omega})}, y_{0}\right)=C_{k}\left(\left.\varphi_{\lambda}\right|_{C_{0}^{1}(\bar{\Omega})}, y_{0}\right) \text { for all } k \geq 0
$$

(see (6.2)). But from Palais [31] (see also Chang [11], p.14) we have

$$
\begin{aligned}
& C_{k}\left(\left.\widehat{\psi}_{\lambda}\right|_{C_{0}^{1}(\bar{\Omega})}, y_{0}\right)=C_{k}\left(\widehat{\psi}_{\lambda}, y_{0}\right), \\
& C_{k}\left(\left.\varphi_{\lambda}\right|_{C_{0}^{1}(\bar{\Omega})}, y_{0}\right)=C_{k}\left(\varphi_{\lambda}, y_{0}\right) \forall k \geq 0 .
\end{aligned}
$$

From (6.3) and (6.4) it follows that

$$
C_{k}\left(\widehat{\psi}_{\lambda}, y_{0}\right)=C_{k}\left(\varphi_{\lambda}, y_{0}\right) \text { for all } k \geq 0
$$

Then from (5.16) and (6.5) it follows that

$$
C_{1}\left(\varphi_{\lambda}, y_{0}\right) \neq 0
$$

Suppose that the spectrum of $\varphi_{\lambda}^{\prime \prime}\left(y_{0}\right)$ is in $[0, \infty)$. Then

$$
\begin{aligned}
& \int_{\Omega}\left(\nabla_{y} a\left(z, D y_{0}\right) D v(z), D v(z)\right)_{\mathbb{R}^{N}} d z \\
\geq & \int_{\Omega} f_{x}^{\prime}\left(z, y_{0}(z)\right) v(z)^{2} d z \text { for all } v \in H_{0}^{1}(\Omega) .
\end{aligned}
$$

If $u \in \operatorname{Ker} \varphi_{\lambda}^{\prime \prime}\left(y_{0}\right)$, then

$$
-\operatorname{div}\left(\nabla_{y} a\left(z, D y_{0}\right) D u(z)\right)=f_{x}^{\prime}\left(z, y_{0}(z)\right) u(z) \text { in } \Omega,\left.u\right|_{\partial \Omega}=0,
$$

hence 


$$
\operatorname{dim} \varphi_{\lambda}^{\prime \prime}\left(y_{0}\right) \leq 1(\operatorname{see}(6.7) \text { and }([20])
$$

therefore

$$
C_{k}\left(\varphi_{\lambda}, y_{0}\right)=\delta_{k, 1} \mathbb{Z} \text { for all } k \geq 0
$$

(see (6.6) and Proposition 2.5 of Bartsch [7]). Recall that $u_{0} \in$ int $C_{+}$is a critical point of mountain pass type of $\varphi_{\lambda}^{+}$. Since

$$
\left.\varphi_{+}^{\lambda}\right|_{C_{+}}=\left.\varphi_{\lambda}\right|_{C_{+}}
$$

as above, we show that

$$
C_{k}\left(\varphi_{\lambda}, u_{0}\right)=\delta_{k, 1} \mathbb{Z} \text { for all } k \geq 0
$$

In a similar fashion, we have

$$
C_{k}\left(\varphi_{\lambda}, v_{0}\right)=\delta_{k, 1} \mathbb{Z} \text { for all } k \geq 0
$$

Moreover, from Proposition 7, we know that $\widehat{u} \in$ int $C_{+}$and $\widehat{v} \in-i n t C_{+}$are local minimizers of $\varphi_{\lambda}$. Hence

$$
C_{k}\left(\varphi_{\lambda}, \widehat{u}\right)=C_{k}\left(\varphi_{\lambda}, \widehat{v}\right)=\delta_{k, 0} \mathbb{Z} \text { for all } k \geq 0
$$

Since $C_{k}\left(\widehat{\psi}_{\lambda}, 0\right)=C_{k}\left(\varphi_{\lambda}, 0\right)$ for all $k \geq 0$, from (5.17) we have

$$
C_{k}\left(\varphi_{\lambda}, 0\right)=0 \text { for all } k \geq 0
$$

Finally, from Proposition 5 of Aizicovici-Papageorgiou-Staicu [3], we have

$$
C_{k}\left(\varphi_{\lambda}, \infty\right)=0 \text { for all } k \geq 0 \text {. }
$$

Suppose $K_{\varphi_{\lambda}}=\left\{0, u_{0}, \widehat{u}, v_{0}, \widehat{v}, y_{0}\right\}$. Then from $(6.8)-(6.13)$ and the Morse relation with $t=-1$, we have

$$
2(-1)^{0}+3(-1)^{1}=0
$$

a contradiction. So we can find $\widehat{y} \in K_{\varphi_{\lambda}}, \widehat{y} \notin\left\{0, u_{0}, \widehat{u}, v_{0}, \widehat{v}, y_{0}\right\}$. Then $\widehat{y}$ is a solution of $\left(P_{\lambda}\right)$ and $\widehat{y} \in C_{0}^{1}(\bar{\Omega})$.

REMARK. It is an interesting open question whether $\widehat{y}$ is nodal or not.

Acknowledgements. The authors wish to thank an anonymous referee for his/her corrections and remarks. The third author gratefully acknowledges the partial support by FEDER funds through COMPETE - Operational Programme Factors of Competitiveness and by Portuguese funds through the Center for Research and Development in Mathematics and Applications and the Portuguese Foundation for Science and Technology (FCT), within project PEst-C/MAT/UI4106/2011 with COMPETE number FCOMP-01-0124-FEDER-022690. 


\section{REFERENCES}

[1] S. Aizicovici, N. S. Papageorgiou, and V. Staicu, Degree theory for operators of monotone type and nonlinear elliptic equations with inequality constraints, Mem. Amer. Math. Soc., 196 (2008).

[2] S. Aizicovici, N. S. Papageorgiou, and V. Staicu, Existence of multiple solutions with precise sign information for superlinear Neumann problems, Ann. Mat. Pura Appl., 188 (2009), pp. 679-719.

[3] S. Aizicovici, N. S. Papageorgiou, And V. Staicu, On a p-superlinear Neumann p-Laplacian equation, Topol. Methods Nonlinear Anal., 34 (2009), pp. 111-130.

[4] S. Aizicovici, N. S. Papageorgiou, and V. Staicu, On p-superlinear equations with a nonhomogeneous differential operator, NoDEA - Nonlinear Differential Equations Appl., 4 (2013), pp. 151-175.

[5] S. Aizicovici, N. S. Papageorgiou, and V. Staicu, Nodal and multiple solutions for nonlinear periodic problems with competing nonlinearities, Commun. Contemp. Math., pp. 1-29, published online 12/03/2013. DOI:10.1142/S0219199713500016.

[6] A. Ambrosetti, H. Brezis, And G. Cerami, Combined effects of concave and convex nonlinearities in some elliptic problems, J. Funct. Anal., 122 (1994), pp. 519-543.

[7] T. Bartsch. Critical point theory on partially ordered Hilbert spaces. J. Funct. Anal., 186:117152, 2001.

[8] V. Benci, D. Fortunato, And L. Pisani, Solitons like solutions of a Lorentz invariant equation in dimension 3, Rev. Math. Phys., 10 (1998), pp. 315-344.

[9] R. Benguria, H. Brezis, And E. H. Lieb, The Thomas-Fermi-von Weizsacker theory of atoms and molecules, Comm. Math. Phys., 79 (1981), pp. 167-180.

[10] H. Brézis and L. Nirenberg, $H^{1}$ versus $C^{1}$ local minimizers, C. R. Math. Acad. Sci. Paris, t. 317 (1993), pp. 465-472.

[11] K. C. ChAng, Infinite Dimensional Morse Theory and Multiple Solution Problems, Birkhauser, Boston, 1993.

[12] S. Cingolani and M. Degiovanni, Nontrivial solutions for p-Laplace equations with right hand side having p-linear growth at infinity, Comm. Partial Differential Equations, 30 (2005), pp. 1191-1203.

[13] D. G. Costa And C. A. Magalhães, Existence results for perturbations of the p-Laplacian, Nonlinear Anal., 24 (1995), pp. 409-418.

[14] M. CUESTA AND P. TAKAC, A strong comparison principle for positive solutions of degenerate elliptic equations, Differential Integral Equations, 13 (2000), pp. 721-746.

[15] J. I. DiAZ AND J. E. SAA, Existence et unicité de solutions positives pour certaines equations elliptiques quasilineaires, C. R. Math. Acad. Sci. Paris, 305 (1987), pp. 521-524.

[16] N. Dunford And J. T. Schwartz, Linear Operators, Part. I. Interscience, New York, 1958.

[17] G. FEI, On periodic solutions of superquadratic Hamiltonian systems, Electron. J. Differential Equations, 2002 (2002), pp. 1-12.

[18] G. Figueiredo, Existence of positive solutions for a class of $p$ \& $q$ elliptic problems with critical growth on $\mathbb{R}^{N}$, J. Math. Anal. Appl., 378 (2011), pp. 507-518.

[19] J. Garcia Azorero, J. Manfredi, and I. Peral Alonso, Sobolev versus Holder local minimizers and global multiplicity for some quasilinear elliptic equations, Commun. Contemp. Math., 2 (2000), pp. 385-404.

[20] L. Gasinski and N. S. PApageorgiou, Nonlinear Analysis, Chapman \&Hall/ CRC Press, Boca Raton, 2006.

[21] Z. Guo And Z. Zhang, $W^{1, p}$ versus $C^{1}$ local minimizers and multiplicity results for quasilinear elliptic equations, J. Math. Anal. Appl., 286 (2003), pp. 32-50.

[22] S. Heikkila and V. Lakshmikantham, Monotone Iterative Techniques for Discontinuous Nonlinear Differential Equations, Marcel Dekker, New York, 1994.

[23] S. Hu AND N. S. PAPAgeOrgiou, Multiplicity of solutions for parametric p-Laplacian equations with nonlinearity concave near the origin, Tohoku Math. J., 62 (2010), pp. 137-162.

[24] Q. JiU AND J. Su, Existence and multiplicity results for Dirichlet problems with p-Laplacian, J. Math. Anal. Appl., 281 (2003), pp. 587-601.

[25] S. Kyritsi AND N. S. PAPAGEOrgiou, Pairs of positive solutions for p-Laplacian equations with combined nonlinearities, Commun. Pure Appl. Anal., 8 (2009), pp. 1031-1051.

[26] O. Ladyzhenskaya and N. Uraltseva, Linear and Quasilinear Elliptic Equations, Academic Press, New York, 1968.

[27] S. Li, S. Wu, AND H. ZHou, Solutions to semilinear elliptic problems with combined nonlinearities, J. Differential Equations, 185 (2002), pp. 200-224.

[28] G. Lieberman, The natural generalization of the natural conditions of Ladyzhenskaya and 
Uraltseva for elliptic equations, Comm. Partial Differential Equations, 16 (1991), pp. 311361.

[29] S. Marano And S. N. S. Papageorgiou, Multiple solutions to a Dirichlet problem with pLaplacian and nonlinearity depending on a parameter, Adv. Nonlinear Anal., 1 (2012), pp. $257-275$.

[30] E. Medeiros And K. Perera, Multiplicity of solutions for a quasilinear elliptic problem via the cohomological index, Nonlinear Anal., 71 (2009), pp. 3654-3660.

[31] R. PAlais, Homotopy theory of infinite dimensional manifolds, Topology, 5 (1966), pp. 1-16.

[32] N. S. Papageorgiou and V. Radulescu, Bifurcation near the origin for the Robin problen with concave-convex nonlinearities, C. R. Math. Acad. Sci. Paris, 352 (2014), pp. 627-632.

[33] N. S. Papageorgiou, E. M. Rocha, and V. Staicu, A multiplicity theorem for hemivariational inequalities with a $p$-L aplacian-like differential operator, Nonlinear Anal., 69 (2008), pp. 1150-1163. 